\title{
Inhibition of vascular adhesion protein 1 protects dopamine neurons from the effects of acute inflammation and restores habit learning in the striatum
}

\author{
Serena Becchi ${ }^{1}$, Alberto Buson ${ }^{2}$ and Bernard W. Balleine ${ }^{1^{*}}$ (D)
}

\begin{abstract}
Background: Changes in dopaminergic neural function can be induced by an acute inflammatory state that, by altering the integrity of the neurovasculature, induces neuronal stress, cell death and causes functional deficits. Effectively blocking these effects of inflammation could, therefore, reduce both neuronal and functional decline. To test this hypothesis, we inhibited vascular adhesion protein 1 (VAP-1), a membrane-bound protein expressed on the endothelial cell surface, that mediates leukocyte extravasation and induces oxidative stress.

Method: We induced dopaminergic neuronal loss by infusing lipopolysaccharide (LPS) directly into the substantia nigra (SN) in rats and administered the VAP-1 inhibitor, PXS-4681A, daily.

Results: LPS produced: an acute inflammatory response, the loss of dopaminergic neurons in the SN, reduced the dopaminergic projection to SN target regions, particularly the dorsolateral striatum (DLS), and a deficit in habit learning, a key function of the DLS. In an attempt to protect SN neurons from this inflammatory response we found that VAP-1 inhibition not only reduced neutrophil infiltration in the SN and striatum, but also reduced the associated striatal microglia and astrocyte response. We found VAP-1 inhibition protected dopamine neurons in the SN, their projections to the striatum and promoted the functional recovery of habit learning. Thus, we reversed the loss of habitual actions, a function usually dependent on dopamine release in DLS and sensitive to striatal dysfunction.
\end{abstract}

Conclusions: We establish, therefore, that VAP-1 inhibition has an anti-inflammatory profile that may be beneficial in the treatment of dopamine neuron dysfunction caused by an acute inflammatory state in the brain.

Keywords: VAP-1, Dopamine neurons, Inflammation, Dorsolateral striatum, Blood-brain barrier, Habit learning

\section{Background}

Many acute and chronic neurodegenerative conditions are accompanied by an inflammatory state in the brain and yet how such changes induce neuronal effects is currently unclear $[1,2]$. One important factor is the influence of brain inflammation on the integrity of the

\footnotetext{
*Correspondence: bernard.balleine@unsw.edu.au

${ }^{1}$ Decision Neuroscience Lab, School of Psychology, UNSW Sydney, Randwick, NSW 2052, Australia

Full list of author information is available at the end of the article
}

neurovasculature generally and the blood-brain barrier $(\mathrm{BBB})$ in particular $[3,4]$. An intact BBB is necessary for tissue homeostasis and to avoid collateral tissue damage whereas a dysfunctional BBB has been linked to various neurological disorders [5] and neurodegenerative diseases [6]. Under inflammatory conditions, the endothelium increases the expression and/or function of adhesion molecules and such endothelial activation has been described in both acute dysfunction [7] and in chronic neurodegenerative diseases such as 
Alzheimer's disease [8-10] and Parkinson's disease [11$13]$ in humans, and in animal models of Parkinson's disease [14-16].

Adhesion proteins control leukocyte migration from the blood stream to the tissue and each step of this migration event is regulated by specific adhesion molecules both on leukocytes and endothelial cells, which increase during inflammation [17]. Vascular adhesion protein 1 (VAP-1) is amongst these adhesion proteins. It is expressed on endothelial cells and binds leukocytes through its semicarbazide-sensitive amine oxidase activity (SSAO) [18-21]. It is key for neutrophil extravasation in vivo and, when the function of VAP- 1 is inhibited, the net effect is the impairment of neutrophil migration into areas of inflammation [22].

As an enzyme, VAP-1 metabolizes primary amines, generating the corresponding aldehydes, hydrogen peroxide $\left(\mathrm{H}_{2} \mathrm{O}_{2}\right)$ and ammonia, which are able to induce cellular damage when and where they are overproduced [23]. Under normal conditions VAP-1 is mainly intracellular, but under inflammatory stimulation it is expressed at the cell membrane of activated endothelial cells and is shed into the blood stream, increasing VAP-1 levels in the blood that can serve as a biomarker for inflammation [24]. VAP-1 expression is also altered in a number of human neuropathologies and animal models [25-32], common features of which are gliosis and leukocyte infiltration $[11,33]$ as well as the microvascular proliferation [12] that can precede and worsen vascular damage and neuronal loss.

The adhesive function of VAP- 1 can be inhibited either by monoclonal antibodies or by small-molecule SSAO inhibitors [34] and, indeed, we have recently shown that the selective VAP-1 inhibitor, PXS-4681A [35], reverses the effects of systemic or ICV lipopolysaccharide (LPS) on microglia activation and neutrophil infiltration in the substantia nigra (SN) and striatum [36]. Here, we infused LPS into the SN to induce an acute inflammatory state sufficient to deplete dopaminergic (DA) neurons [37, 38] and attempted to block this effect with a VAP-1 inhibitor and so rescue DA neuronal loss, the loss of the DA projection in the striatum and associated functional deficits particularly in habit learning, a critical psychological function commonly lost in both acute and chronic conditions that interfere with neural function [39].

\section{Materials and methods}

\section{Animals}

Hooded Wistar male rats between 300 and 400 g obtained from Adelaide Laboratory Animal Services (University of Adelaide, Australia) were used in these experiments. Animals were housed in groups of up to 3-4 per cage in a $12 / 12 \mathrm{~h}$ light/dark cycle (7:00AM to 7:00PM) with full access to food and water and environmental enrichment. Throughout the behavioural experiment, rats were maintained at $\sim 85 \%$ of their free-feeding body weight by restricting their food intake. All animals were humanely killed via injection of pentobarbitone sodium in compliance with the ARRIVE guidelines [40-42].

\section{Drugs and drug treatments}

Unless otherwise specified, chemicals reagents and solvents were purchased from Sigma Aldrich (St. Louis, $\mathrm{MO})$. The VAP-1 inhibitor PXS-4681A, [(Z)-4-(2(aminomethyl)-3fluoroallyloxy)benzenesulfonamide hydrochloride], was synthesized by Pharmaxis Ltd [35].

\section{Intranigral LPS lesion}

Before the start of surgery, the animals received injections of antibiotic, Benacillin (Ilium) and the local anaesthetic Bupivacaine (Hospira) injected subcutaneously (sc) at the surgical site. Rats were anaesthetized with isoflurane (5\% for induction and $2-3 \%$ for maintenance) and positioned in a stereotaxic frame (Stoelting, Wood Dale, IL, USA). An incision was made to expose the scalp and the incisor bar was adjusted to align bregma and lambda on the same horizontal plane.

\section{Unilateral infusions}

For all rats, holes were drilled into the skull at the following coordinates: $-5.3 \mathrm{~mm}$ posterior; $\pm 2.0 \mathrm{~mm}$ lateral; $-8.0 \mathrm{~mm}$ ventral to bregma [43]. Each unilateral injection of LPS from Escherichia coli (O55:B5) $(2 \mu \mathrm{l}$ of $1.5 \mathrm{mg} / \mathrm{ml}$ solution of LPS) into the SN was conducted using a glass capillary filled onto a Nanoject II (Drummond Scientific) mounted on the stereotaxic frame in order to minimize the mechanical damage of the needle to the tissue. Once in position, LPS was delivered at an approximate rate of $100 \mathrm{~nL} / \mathrm{min}$, plus an additional 10 min where the needle was left in situ to avoid reflux along the injection track. Warm saline $(5 \mathrm{ml})$ was administered at the end of the surgical procedure.

\section{Bilateral infusions}

The procedures were identical as described for unilateral infusions. LPS was infused into the lateral SN at the following coordinates: $-5.3 \mathrm{~mm}$ posterior; $\pm 2.7 \mathrm{~mm}$ lateral; $-7.4 \mathrm{~mm}$ ventral to bregma [43], whereas a saline control group received bilateral injections of sterile saline into the lateral SN.

\section{PXS-4681A treatment}

$1 \mathrm{~h}$ before and $5 \mathrm{~h}$ after LPS infusions, animals received $2 \mathrm{mg} / \mathrm{kg}$ of PXS-4681A dissolved in phosphate buffer saline (PBS), ip. Once a day injection was continued for the remainder of the experiment. 


\section{Immunohistochemical procedures}

At day 16 the animals were killed with pentobarbital sodium and transcardially perfused with $400 \mathrm{ml}$ PBS at $4{ }^{\circ} \mathrm{C}$ followed by $400 \mathrm{ml}$ of $4 \%$ paraformaldehyde (PFA) solution at $4{ }^{\circ} \mathrm{C}$. Brains were fixed in the same fixative before being sectioned with a Leica VT 1000S Vibratome (Leica Microsystem). Coronal sections of $30 \mu \mathrm{m}$ containing the $\mathrm{SN}$ or the striatum were washed $3 \times 0.1 \mathrm{M}$ at $\mathrm{pH}$ 7.4 PBS for $10 \mathrm{~min}$.

\section{Dopaminergic neurons}

DA neurons were stained with mouse anti-tyrosine hydroxylase $(\mathrm{TH})$ antibody in both $\mathrm{SN}$ and striatum and visualized using nickel-enhanced 3,3'-diaminobenzidine (DAB). After washing the sections in PBS $3 \times 10 \mathrm{~min}$, they were quenched for $10 \mathrm{~min}$ in $10 \%$ methanol and $3 \%$ $\mathrm{H}_{2} \mathrm{O}_{2}$. Sections were then rinsed $3 \times 10 \mathrm{~min}$ in PBS and blocked in blocking solution PBS containing 5\% normal serum and $0.25 \%$ Triton-X-100. The primary antibody was incubated in blocking solution overnight at $4{ }^{\circ} \mathrm{C}$. Sections were washed and incubated in biotin-SP AffiniPure donkey anti-mouse IgG (Jackson ImmunoResearch, USA) for $2 \mathrm{~h}$, they were then incubated with ABC (Avidin-Biotin Complex) Kit (VectorLabs, Australia) for $1 \mathrm{~h}$. DAB development was conducted adding glucose oxidase in $0.1 \mathrm{M}$ acetate buffer $\mathrm{pH}$ 6.0. Sections were mounted and coverslipped with Entellan ${ }^{\circledR}$ (Merk, Australia).

Cell count in the $\mathrm{SN}$ was performed live using an upright microscope (Olympus BX50, Japan) under a $10 \times$ Zeiss LSM 7110 CLSM (Carl Zeiss, Germany) lens. Boundaries of the regions of interest were created using a $10 \times 10$ grid reticule $(1 \times 1 \mathrm{~mm})$ located in the right eyepiece of the microscope. One in every six sections between bregma $-4.80 \mathrm{~mm}$ and $-6.12 \mathrm{~mm}$ [43] was counted manually. Values across sections were averaged for each animal. The operator was blind to treatment condition using a random renumbering of the samples before the beginning of data acquisition.

For the striatum, one in every six sections between bregma $+1.9 \mathrm{~mm}$ and $0.00 \mathrm{~mm} \mathrm{[43]} \mathrm{was} \mathrm{quantified}$ with Open Source ImageJ software (MacBiophotonics upgrade version $1.43 \mathrm{u}$, Wayne Rasband, National Institutes of Health, Bethesda, MD). Images of dorsal striatum were acquired with a $20 \times$ Zeiss LSM CLSM lens using a confocal laser scanning microscope (FV1000, Olympus, Japan) in both ipsilateral and contralateral hemispheres. In each hemisphere, two different ROIs were defined: ROI 1 comprised the grey matter of the dorsal striatum and ROI 2 comprised the fibre bundles with no TH staining and was used as background correction. The mean grey value of $\mathrm{TH}$ staining was collected for each ROIs by ImageJ software, and expressed as ROI 1 - ROI 2. Again, the operator was blind to the groups using the random renumbering of samples before the beginning of the data acquisition by a different experimenter blind to the treatment condition, and the group assignment was not known until all analyses were complete. One grey value was obtained per animal.

Subjects with an inaccurate injection site were excluded from the statistical analysis during post-mortem assessment. Correct injection placements were considered when the needle track was found between -5.10 and - $5.40 \mathrm{~mm}$ posterior to bregma [43] and touching the pars compacta.

\section{Immunofluorescence procedure and quantification}

Unless specified, immunofluorescence staining was conducted as follows: after washing the sections in Trisbuffered saline (TBS) $3 \times 10 \mathrm{~min}$, they were left in the blocking solution for $1 \mathrm{~h}$, followed by incubation in primary antibodies dissolved in blocking buffer overnight at $4{ }^{\circ} \mathrm{C}$. After washing in TBS $3 \times 10 \mathrm{~min}$, they were incubated in secondary antibodies dissolved in blocking solution for $2 \mathrm{~h}$. Sections were then rinsed in TBS $3 \times 10 \mathrm{~min}$, mounted and coverslipped with Vectashield (VectorLab, Australia). Images were acquired with a $20 \times$ Zeiss LSM CLSM lens using a confocal laser scanning microscope (FV1000, Olympus) and intensity of the staining was quantified as mean grey value with Open Source Image software. Refer to Table 1 for dilutions and product numbers of antibodies.

Immunofluorescence on striatal sections was performed on four to six sections chosen every $0.35 \mathrm{~mm}$ from bregma +1.90 to $0.12 \mathrm{~mm}$ [43] and each specific staining was quantified in an area of $635.9 \mu \mathrm{m}$ (h) $\times 635.9 \mu \mathrm{m} \mathrm{(w)} \mathrm{in} \mathrm{both} \mathrm{hemispheres} \mathrm{using} \mathrm{Image} \mathrm{J}$ software.

Myeloperoxidase (MPO) staining was conducted slightly differently: samples were boiled for $6 \mathrm{~min}$ in $10 \mathrm{mM}$ citrate buffer at $\mathrm{pH} 6.0$ before immunostaining procedure. MPO antibody was incubated overnight with ionized calcium binding adaptor molecule (Iba)-1 and rat endothelial cell antibody (RECA)-1, and the combination of the three was used to identify neutrophils and their location relative to the blood vessels [36]. A combination of secondary antibodies (donkey anti-mouse IgG Alexa Fluor $^{\circledR} 405$, donkey anti-rabbit IgG Alexa Fluor ${ }^{\circledR} 488$ and donkey anti-goat IgG Alexa Fluor ${ }^{\circledR}$ 546) and NeuroTrace $^{\mathrm{TM}} 640 / 660$ Nissl was used to visualize the staining. In the $\mathrm{SN}$, images were acquired with $10 \times$ Zeiss LSM 7110 CLSM (Carl Zeiss, Germany) lens at high resolution $(4800 \times 4800$ pixels per image $)$ and neutrophil cells were manually counted using Image J cell count. MPO-positive cells were counted in three sections between bregma $-4.80 \mathrm{~mm}$ and $-5.70 \mathrm{~mm}$ [43] that were $0.3 \mathrm{~mm}$ 
Table 1 List of antibodies (Key resources table)

\begin{tabular}{|c|c|c|}
\hline Reagent or Resource & Source & Identifier \\
\hline \multicolumn{3}{|l|}{ Antibodies } \\
\hline Rabbit anti-glial fibrillary acidic protein & Abcam (1:1000) & AB7260 \\
\hline Rabbit anti-interleukin 1 beta & Santa Cruz & SC-7884 \\
\hline Rat anti-interleukin 10 & Abcam (1:500) & AB33471 \\
\hline Goat anti-ionized calcium binding adapter molecule & Abcam (1:2000) & AB5076 \\
\hline Rabbit anti-ionized calcium binding adapter molecule & Wako $(1: 1000)$ & 019-19741 \\
\hline Rabbit anti-myeloperoxidase & Abcam (1:300) & AB45977 \\
\hline Mouse anti-rat endothelial cell antigen & Serotec $(1: 300)$ & MCA970R \\
\hline Mouse anti-tyrosine hydroxylase & Millipore $(1: 10,000)$ & AB1542 \\
\hline Rabbit anti-tumour necrosis factor alpha & Abcam (1:500) & AB6671 \\
\hline Rabbit anti-vascular adhesion protein 1 & Abcam (1:300) & AB42885 \\
\hline Donkey anti-rat IgG Alexa Fluor ${ }^{\circledR} 594$ & Invitrogen (1:1000) & A21209 \\
\hline Donkey anti-rabbit lgG Alexa Fluor ${ }^{\circledR} 488$ & Invitrogen (1:1000) & A21206 \\
\hline Donkey anti-mouse lgG Alexa Fluor ${ }^{\circledR} 405$ & Invitrogen (1:1000) & A48257 \\
\hline Donkey anti-goat IgG Alexa Fluor ${ }^{\circledR} 546$ & Invitrogen (1:1000) & A11056 \\
\hline NeuroTrace ${ }^{\mathrm{TM}} 640 / 660$ Nissl & Thermofisher (1:1000) & N21483 \\
\hline Donkey anti-goat IgG Alexa Fluor ${ }^{\circledR} 594$ & Invitrogen (1:1000) & A11057 \\
\hline Donkey anti-mouse lgG Alexa Fluor ${ }^{\circledR} 546$ & Invitrogen (1:1000) & A10036 \\
\hline Biotin-SP AffiniPure donkey anti-mouse lgG & Jackson ImmunoResearch (1:400) & $715-065-150$ \\
\hline Mouse anti-major histocompatibility complex II & Abcam (1:500) & AB23990 \\
\hline Mouse anti-postsynaptic density protein 95 & Invitrogen (1:500) & MA1-045 \\
\hline
\end{tabular}

equidistant from the injection site. In the striatum, four sections between bregma +1.90 and $0.12 \mathrm{~mm}$ [43] were counted. One value per animal per hemisphere and area was obtained.

Microglia cells were quantified as Iba1-positive cell number and Iba1-positive area with Image J. Cell count was performed with Image J cell counter plugin while for Iba-1-positive area a fix thresholding was applied to each image and measured.

Double staining for mouse anti-major histocompatibility complex (MHC) II and goat anti-Iba1 was carried as follow. The fluorescence analysis was performed so that a ROI comprised the microglia defined by Iba1positive area. Mean grey intensity values for each marker were obtained quantifying the intensity of the staining of MHC II within the microglia-defined ROI. A single value of mean grey value was obtain in each hemisphere for each marker for each rat. All double stainings were visualized with Alexa Fluor ${ }^{\circledR} 488$ and Alexa Fluor ${ }^{\circledR} 594$ or Alexa Fluor $546^{\circledR}$ conjugated secondary antibodies.

Rabbit anti-glial fibrillary acidic protein (GFAP) was used to label astrocytes and quantified as GFAP-positive area in each image. GFAP-positive area was identified using a fix thresholding parameter using Image J. In the $\mathrm{SN}$, the GFAP-positive area was quantified using an Olympus VS-120 slide scanner. Three ROIs were created in the LPS side and in the contralateral hemisphere in six coronal sections between bregma $-4.80 \mathrm{~mm}$ and $-6.12 \mathrm{~mm}$ [43], every $0.25 \mathrm{~mm}$, covering the $\mathrm{SN}$ area. The average of intensity of staining was calculated with image J from each ROI and averaged per hemisphere and per animal.

VAP-1 expression was revealed with rabbit anti-VAP-1 and co-stained with anti-RECA-1 and revealed with donkey anti-rabbit IgG Alexa Fluor ${ }^{\circledR} 488$ and donkey anti-mouse IgG Alexa Fluor ${ }^{\circledR} 546$ secondary antibodies. NeuroTrace ${ }^{\text {TM }} 640 / 660$ deep red fluorescent Nissl was incubated with secondary antibodies for $1 \mathrm{~h}$ at room temperature.

Post-synaptic puncta were identified with mouse antipost synaptic density (PSD)-95 and visualized with donkey anti-mouse Alexa Fluor ${ }^{\circledR}$ 546. Images of PSD-95 staining were taken with $60 \times$ Zeiss LSM 7110 CLSM (Carl Zeiss, Germany), zoomed $2 \times$. The intensity of PSD-95 staining was quantified in images representing an area of $35 \mu \mathrm{m}(\mathrm{h}) \times 35 \mu \mathrm{m}(\mathrm{w})$ in four sections from bregma +1.90 and $0.12 \mathrm{~mm}$ [43], chosen every $0.45 \mathrm{~mm}$ in the dorsal striatum. A threshold was calculated for each image using Image J software and a mask was created. The values of PSD-95-positive staining as mean grey value and positive area were quantified in each image. Values of four sections were averaged for each hemisphere for each rat and treated as a single $n$. 


\section{Blood-brain barrier leakage}

Breakdown of the blood-brain barrier (BBB) was assessed by employing a one-step immunohistochemical detection of IgG as in Tomás-Carmadiel et al. [44] and Schmidt-Kastner et al. [45]. Briefly, seven sections between bregma $-4.80 \mathrm{~mm}$ and $-6.12 \mathrm{~mm}$ [43] were incubated for $2 \mathrm{~h}$ in donkey anti-rat IgG Alexa Fluor ${ }^{\circledR} 594$ (Invitrogen; 1:1000 in PBS containing 1\% bovine serum albumin and $0.2 \%$ Triton-X-100) and then double-stained with rabbit anti-GFAP, followed by donkey anti-rabbit IgG Alexa Fluor $^{\circledR}$ 488. Visualization of GFAP and IgG immunoreactivity was detected under $4 \times$ Zeiss LSM 7110 CLSM (Carl Zeiss, Germany) and Image J was used to calculate the IgG-positive area per section.

\section{Rotational behavior}

At day 14 animals were tested for spontaneous forelimb akinesia using a cylinder test and for rotational behaviour induced by apomorphine. The cylinder test assesses a rat's ability to use each forelimb to support its body against the wall of a cylindrical enclosure. We performed this test following the procedure reported by Schallert and Tillerson [46]. Briefly, rats were put individually in a glass cylinder $(20 \mathrm{~cm}$ diameter, $30 \mathrm{~cm}$ height) and video recorded for $5 \mathrm{~min}$. No habituation to the cylinder prior to filming was allowed. The test was performed between 10.00 and $14.00 \mathrm{~h}$. Two mirrors were placed to the sides of the cylinder at an angle that enable the recording of forelimb movements even when the animal was turned away from the camera. Scoring was conducted by an experimenter blind to the experimental treatment using VLC software with slow-motion and clear stop-frame capabilities. The behaviour was scored for independent use of the left or right forelimb to contact the cylinder wall during a full rear to initiate a weight-shifting movement or to regain centre of gravity while moving laterally in a vertical posture [46].

Apomorphine-induced rotational behaviour was assessed the day after the cylinder test. Each rat was placed in a circular arena (30 cm diameter) for $5 \mathrm{~min}$ before receiving an ip injection of $0.5 \mathrm{mg} / \mathrm{kg}$ of apomorphine hydrochloride dissolved in $0.02 \%$ ascorbic acid and saline. Rotational behaviour was recorded for $30 \mathrm{~min}$ after apomorphine injection and scored later by an experimenter blind to treatment condition [47].

\section{Instrumental training and testing \\ Apparatus}

All behavioural procedures were performed in 16 identical Med Associates (USA) operant chambers enclosed in sound- and light-attenuating shells. Each chamber was equipped with a pump that was fitted with a syringe that delivered $20 \%$ sucrose solution $(0.1 \mathrm{ml})$ into a recessed food magazine. An infrared photobeam that crossed the magazine allowed for the detection of magazine head entries. Each chamber contained two retractable levers to the right and left of the magazine and a $3 \mathrm{~W} 24 \mathrm{~V}$ house light mounted on the top of the wall opposite the magazine provided illumination. Two microcomputers running on the Med-PC program (Med Associates) controlled experimental events and recorded lever presses and magazine entries.

\section{Lever-press training for habit}

Following 4 days of food deprivation, rats were given two sessions of magazine training. Sucrose solution was delivered at random $60 \mathrm{~s}$ intervals for 30 outcomes per session. Animals then received 8 days of instrumental training (two sessions per day) to press a single lever for sucrose solution delivery. Right and left levers were counterbalanced across animals. Rats initially received three sessions in which the sucrose was delivered on a continuous reinforcement schedule and then four sessions in which it was delivered on a random interval schedule of $15 \mathrm{~s}$ (RI-15), four sessions on a RI-30 schedule, and four sessions on a RI-60 schedule. Each session commenced with the insertion of the lever; sessions ended when 30 reinforcers were earned or after $60 \mathrm{~min}$, whichever came first. All groups received the same total number reinforcers.

\section{Outcome devaluation}

The day after the last session of training, the sucrose solution was devalued using conditioned taste aversion. All rats were given ad libitum access to sucrose solution for 30 min each day for 3 consecutive days in clear plastic tubs with bottles filled with sucrose solution attached. On each day, half of each lesion group received an intraperitoneal injection of lithium chloride $(0.15 \mathrm{M} \mathrm{LiCl}$, $10 \mathrm{ml} / \mathrm{kg}$ ) and were placed back in their home cages, whereas the other half received saline injections $(10 \mathrm{ml} /$ $\mathrm{kg}$ ). During outcome devaluation no PXS-4681A was administered to the rats with the aim to minimize any possible secondary effect of the drug on devaluation. The amount of sucrose solution consumed each day was also measured.

\section{Extinction test}

Over the 2 days following the last day of outcome devaluation, all rats received two extinction tests. The tests began with the insertion of the same lever used during training and ended with the retraction of the lever after $10 \mathrm{~min}$. Lever presses and magazine entries were recorded, but no sucrose reinforcers were delivered. 


\section{Lever-press training for goal-directed learning}

After conditioned taste aversion devaluation and tests, a sub-cohort of rats was trained to receive two different types of outcome, purified pellets and grain pellets. One outcome was earned for pressing the left lever and the other for pressing the right lever. The identity of the lever-outcome relationships was counterbalanced across subjects.

Twenty outcomes on each lever were delivered per session. Rats received 10 days of instrumental training, during which time the left and right lever press responses were trained with the 2 different outcomes in separate sessions each day. The interval between the training sessions for each lever press action was $10 \mathrm{~min}$. The order of the lever presentations was alternated and counterbalanced across rats and days. The rats received 2 days in which lever pressing was continuously reinforced (CRF); then, the probability of the outcome given a response was gradually shifted over days using increasing random ratio (RR) schedules; a RR5 schedule (probability of receiving an outcome given a response $=0.2$ ) and a RR10 (probability of receiving an outcome given a response $=0.1$ ). Rats received 3 RR 5 sessions followed by 5 RR10 sessions.

\section{Specific satiety outcome devaluation}

Twenty-four hours after the final instrumental training session, rats were given an outcome devaluation test. In this test, rats received ad libitum access to 1 of the 2 outcomes for $1 \mathrm{~h}$ in clear plastic feeding cages. Half of the rats in each response-outcome assignment received grain pellets and the remaining rats received purified pellets. Immediately after devaluation, rats were given a 10-min choice extinction test in which both levers were available but no outcome was delivered. Testing in extinction ensured that the rats had to recall both the current action-outcome contingencies and the current values of the two instrumental outcomes to choose appropriately. The following day all rats were given a second test with the other outcome devalued; that is, rats that were prefed on grain pellets for the first devaluation test were now pre-fed with purified pellets and vice versa.

\section{Locomotor assessment with rotarod and grip strength meter}

At the end of instrumental testing, motor balance and coordination were evaluated with a rotarod (Ugo Basile, Italy). Rats were trained on the rotating rod at a fixed speed of $4 \mathrm{rpm}$ for 3 trials until they stayed on the rod for at least $2 \mathrm{~min}$. On the following days, the speed increased from 4 to $50 \mathrm{rpm}$ within $5 \mathrm{~min}$. Each animal had three trials of testing on two separate days with a resting period of at least $2 \mathrm{~min}$ between each trial. The time the animal spent on the rotarod was measured as latency to fall and the average of the six trials was considered per rat.

Forelimb grip strength was measured after the rotarod test as maximum tensile force using a rat grip strength meter (Columbus Instruments, Columbus, USA) with a sensor range of 0-5,000 g, and accuracy of $0.15 \%$. Ten trials per rat were recorded and the average of the highest 5 pulls of the grip strength meter was recorded for each animal.

\section{Statistical analysis}

The data are expressed as mean \pm SEM. For statistical significance, experiments with two groups were analysed using two-tailed $t$-tests (unpaired). Experiments with more than two groups were analysed using oneway or two-way ANOVA or repeated measures two-way ANOVA, as appropriate, followed by Sidak's tests for multiple comparisons when an interaction was found to be significant. A value of $p<0.05$ was considered statistically significant. All data were analysed using Prism 8 for Mac OS X, Version 8.0 (Graph Pad software).

\section{Results}

VAP-1 inhibition protects the BBB after LPS stimulus

We have shown previously that ICV inflammation increases endothelial VAP-1 expression [36]. To confirm that local inflammation also induces measurable VAP-1 expression in the BBB, 24 $\mathrm{h}$ after local LPS injection into $\mathrm{SN}$ (Fig. 1A), the membrane-bound form of VAP-1 was found on the endothelial cells of the BBB but not in the parenchyma of the brain (Fig. 1B-D). We also observed strong VAP-1 expression on the choroid plexus in the brain ventricles, which has not previously been reported (Fig. 1E).

Because damage or leakage of the $\mathrm{BBB}$ can cause infiltration of immune cells that can lead to neuronal dysregulation and, ultimately, degeneration $[11,15$, 48-50], we first assessed the role of VAP-1 in BBB damage induced by LPS infusion. Breakdown of the BBB was assessed by immunohistochemical detection of IgG extravasation [44]. Twenty-four hours after LPS there was massive infiltration of anti-rat IgG in the brain parenchyma (Fig. 1F-H) (LPS effect $F_{(1,10)}=91.38$, $p<0.0001)$. VAP-1 enzyme activity was completely inhibited by ip injection of specific small-molecule inhibitor, PXS-4681A [35].

PXS-4681A prevented IgG infiltration suggesting that it protected $\mathrm{BBB}$ integrity: in contrast to the significant difference between the drug- and vehicle-treated LPS hemispheres (LPS-Vehicle vs. LPS-PXS-4681A), $p=0.014$, there was no difference between drug- and 


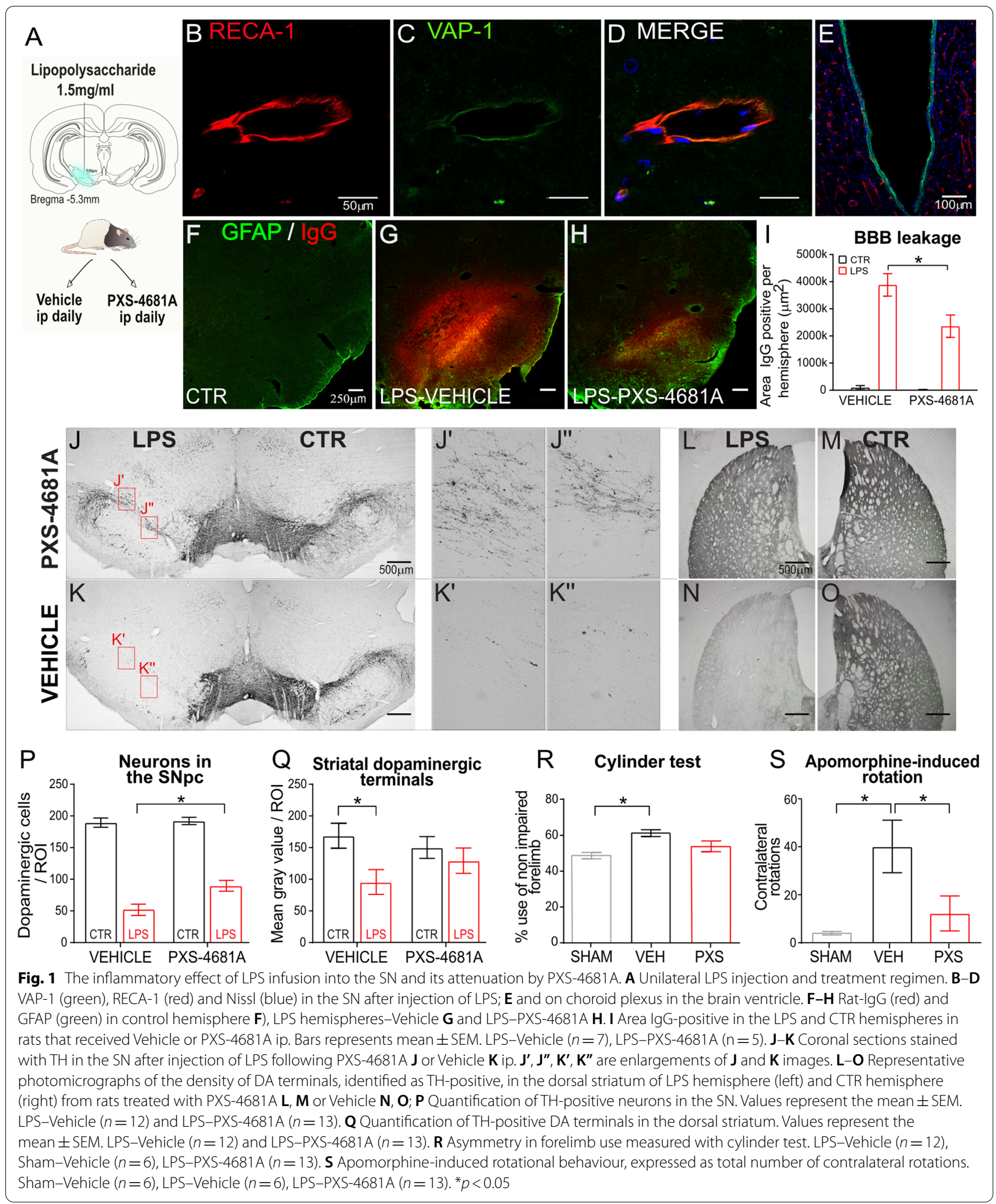


vehicle-treated control hemispheres, producing a significant group $\mathrm{x}$ treatment interaction (repeated measures two-way ANOVA showed an effect of LPS, $F_{(1,10)}=91.38$, $p<0.0001$, no effect of PXS-4681A effect $F_{(1,10)}=4.160$, but a significant interaction $F_{(1,10)}=5.045, p=0.0485$ (Fig. 1I).

\section{VAP-1 inhibition protects dopaminergic neurons after LPS stimulus}

We next quantified DA cells 2 weeks after LPS infusion $[38,51]$, by manual count of $\mathrm{TH}$-positive cells in the $\mathrm{SN}$ and $\mathrm{TH}$-positive projections in the striatum, as the most commonly used marker for DA neurons.

TH in LPS-vehicle and PXS-4681A-treated animals differed significantly (Fig. 1J, K). The number of cells on the contralateral side was comparable in animals treated with PXS-4681A or vehicle indicating that LPS action was localized. In vehicle-treated rats, the number of $\mathrm{TH}$-positive cells was $28 \% \pm 4.79 \%$, whereas in PXS4681A-treated animals more cells survived, $46 \% \pm 3.6 \%$ of the contralateral side; ANOVA showed a main effect of LPS, $F_{(1,23)}=365.22$, a main effect of PXS-4681A $F_{(1 \text {, }}$ ${ }_{23)}=5.012, p=0.0351$ and an interaction between these variables, $F_{(1,23)}=7.695, p=0.011$. Sidak's multiple comparison revealed a significant effect in the LPS hemisphere, LPS-Vehicle $v s$. LPS-PXS-4681A, $p=0.003$, but no difference in the control hemispheres (Fig. 1P).

DA neurons in the SN pars compacta project to the dorsal striatum and, therefore, the cell loss in $\mathrm{SN}$ caused a reduction of $\mathrm{TH}$-positive staining in this projection area. This reduction was significantly diminished by PXS4681A (Fig. 1L-O). The area of TH-positive staining contralateral to the LPS infusion did not differ whether the rat received PXS-4681A or vehicle, again confirming the localized action of LPS and the absence of an effect of the VAP-1 inhibitor on healthy tissue. On the ipsilateral side, vehicle-treated rats showed significantly lower TH-positive terminals $(58.7 \% \pm 7.51 \%)$ than PXS-4681A-treated animals $(81.7 \pm 4.89 \%)$ indicating a prominent protective effect of the drug. Repeated measures two-way ANOVA showed an effect of LPS, $\mathrm{F}_{(1,23)}=25.06, p<0.0001$, no effect of PXS-4681A, $F_{(1,23)}=0.089$, but a significant LPS x PXS-4681A interaction, $F_{(1,23)}=7.857, p=0.01$. Sidak's multiple comparisons showed a significant difference between the LPS vs. control hemisphere in the Vehicle group $(p<0.0001)$ but no difference between hemispheres in PXS-4681A group (Fig. 1Q).

\section{VAP-1 inhibition induces motor recovery in LPS-lesioned rats}

Unilateral DA neuron loss causes motor asymmetry and the effect of this loss was assessed by cylinder and apomorphine-induced rotation tests [52]. Cylinder test showed an increase in the use of the non-impaired forepaw, indicating a difference in level of dopamine between the two hemispheres, which was greater in the vehicle-treated LPS-lesioned group; i.e. they supported their bodies on the wall using the ipsilateral or nonimpaired forelimbs $63.8 \%$ of the time. We first confirmed that the data satisfied both assumptions of parametric tests: test of normal distribution, Kolmogorov-Smirnov and Shapiro-Wilk, $p>0.05$; and homogeneity of variance, Brown-Forsythe test and Bartlett's test, $p>0.05$. Subsequently, one-way ANOVA revealed a group difference $F_{(2,28)}=4.493, p=0.0203$. Sidak's multiple comparisons then indicated Groups LPS-Vehicle significantly increased the ipsilateral forelimb use as compared to Sham group, $p=0.03$. The group receiving PXS-4681A, on the other hand, showed a more even forepaw usage, reaching $55.4 \%$ use of the non-impaired forelimb, which was similar to the sham group, $51.3 \%(p>0.05)$ (Fig. 1R).

Both cylinder test and apomorphine-induced rotation measure the asymmetric depletion of DA projections in the striatum [53], but they differ in the molecular mechanisms that induce stereotypical movements. Forelimb use in cylinder test is dependent on the DA activity in the striatum $[54,46]$ assessing the independent use of each forelimb in the context of a naturally occurring behaviour, whereas apomorphine stimulates D2 and D3 postsynaptic receptors, inducing abnormal contralateral rotations in hemiparkinsonian rats $[55,56]$. In this specific experiment, we avoided to use amphetamine as its molecular structure is similar to the VAP-1 substrate, benzylamine, and that may cause interference with the rotational measurement.

Although we saw a reduction of DA neurons of about $70 \%$ in the SN and $40 \%$ in the striatum, apomorphineinduced turning behaviour revealed an overall low stereotypical rotation. However, as with the cylinder test, the apomorphine test showed a clear difference between groups, $F_{(2,14)}=6, p=0.0093$. Specifically, LPS-lesioned rats showed significantly higher rotation counts than sham rats $(p=0.010)$. The LPS-PXS-4681A group displayed low rotational activity, comparable to the values measured in the sham group $(p>0.05)$ and the LPS-PXS$4681 \mathrm{~A}$ and LPS-Vehicle groups differed significantly $(p=0.0497)$ (Fig. 1S).

\section{VAP-1 inhibition reduces LPS-induced inflammation in the substantia nigra}

Following brain insult astrocytes react inducing astrogliosis $[57,58]$, which involves increased expression of the protein GFAP [59]. In normal conditions, GFAP staining in the SN is very low and mainly localized in the SN pars reticulata [60]. In our condition, GFAP immunofluorescence was increased in the SN after LPS 

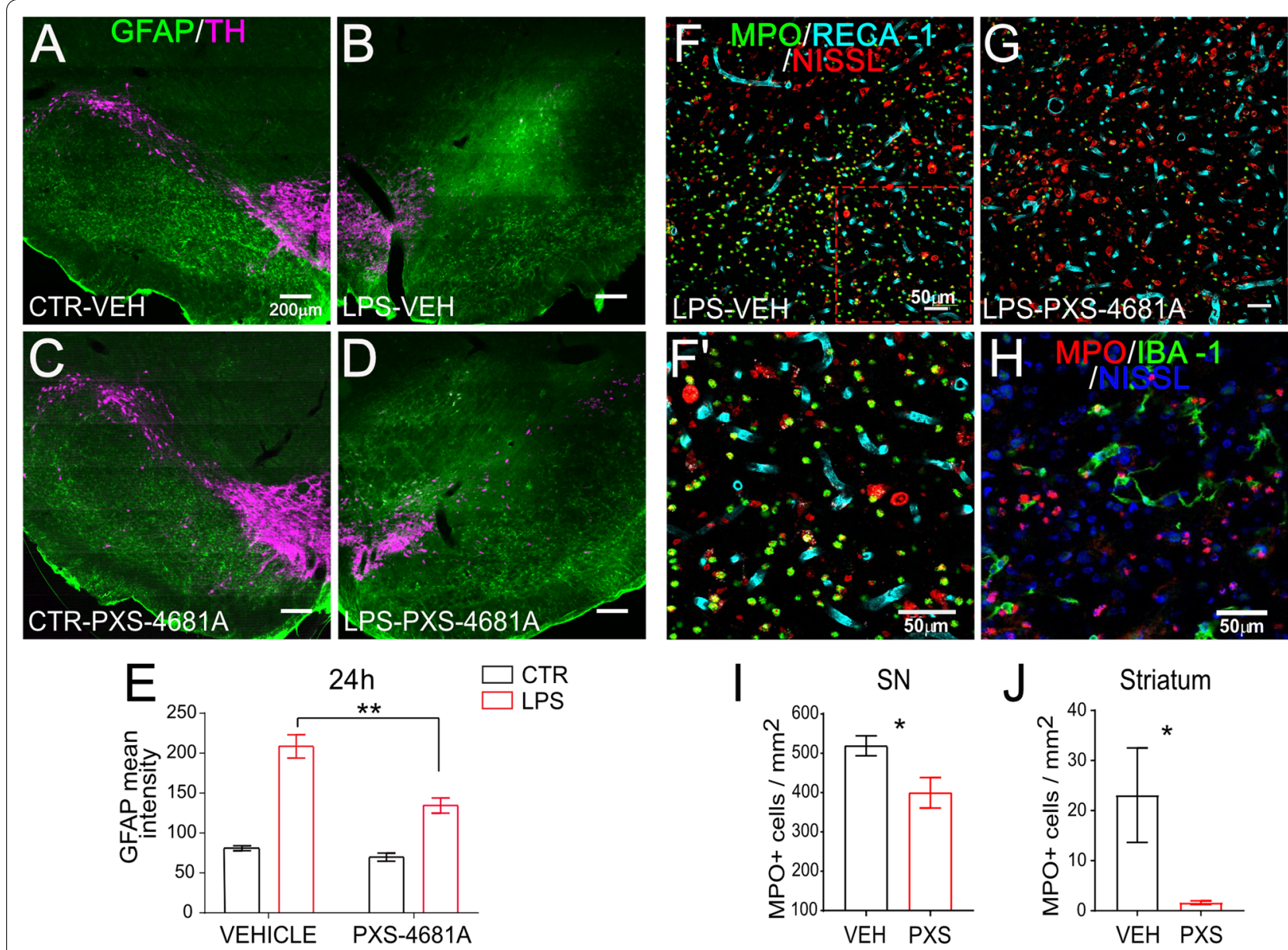

Fig. 2 VAP-1 inhibition reduces the inflammatory response in the SN. A-D Coronal brain sections of SN immunostained for GFAP and TH revealed an increased GFAP intensity in the LPS hemispheres ( $\mathbf{B}$ and $\mathbf{D}$ ) as compared to CTR hemispheres (A and $\mathbf{C})$. E Quantification of GFAP intensity in the SN at $24 \mathrm{~h}$ from LPS insult. Values represent the mean \pm SEM. LPS-Vehicle $(n=7)$, LPS-PXS-4681A $(n=5)$. F, G Immunostaining for MPO, RECA-1-positive blood vessels and Nissl in the SN $24 \mathrm{~h}$ after LPS insult. $\mathbf{F}^{\prime}$ Enlargement of F: MPO-positive cells mainly localized outside blood vessels. $\mathbf{H}$ Double immunostaining for MPO and Iba1 revealed a low percentage of colocalization, indicating that MPO-positive cells were mainly neutrophils. I, J Quantification of MPO-positive cells in the SN that received LPS I and in the ipsilateral striatum $\mathbf{J}$ at $24 \mathrm{~h}$. Values represent the mean \pm SEM. LPS-Vehicle $(n=7)$, LPS-PXS-4681A $(n=5) .{ }^{*} p<0.05 ;{ }^{* *} p<0.01$

infusion, corresponding to the area with high IgG signalling where the $\mathrm{BBB}$ had lost integrity (Fig. 1F-H); effect of LPS, $F_{(1,10)}=41.08, p<0.0001$ (Fig. 2A-D). VAP-1 inhibition decreased the LPS-induced response towards the levels in the control hemisphere (Fig. 2E) producing an effect of PXS-4681A, $F_{(1,10)}=6.155, p=0.0325$ and an LPS x PXS-4681A interaction, $F_{(1,10)}=5.545, p=0.0403$. Sidak's multiple comparisons revealed a difference in the effect of PXS-4681A on GFAP intensity in the LPS treated hemispheres (LPS-Vehicle vs. LPS-PXS-4681A, $p=0.0055$ ) but no effect in the control hemispheres.

Based on its properties as a VAP-1 inhibitor, we assessed the ability of PXS-4681A to control the leukocyte response in the SN. At 24-h post-lesion, there was a substantial accumulation of MPO-positive cells in the lesioned SN in both vehicle and PXS-4681A-treated rats (Fig. 2F, G), whereas this response was absent in the control hemispheres.

LPS challenge disrupts the BBB, and it is conceivable that the leakage of the vessel walls and the loss of tight junctions could cause unregulated entry of blood cells into the brain parenchyma. Most of the inflammatory cells infiltrating the SN were MPO-positive/Iba1-negative neutrophils (Fig. 2H), and as shown by co-labelling with RECA-1 antibody, MPO-positive cells were mostly localized within the tissue after BBB extravasation. Blocking VAP-1 was able to reduce the infiltration of leukocytes, as seen in the SN after PXS-4681A as compared to the LPS-Vehicle group ( $t$-test, $p=0.012)$ (Fig. 2I). 


\section{VAP-1 inhibition reduces the LPS-induced inflammatory response in the striatum}

The intranigral injection of LPS was accompanied by an inflammatory response in the striatum, already visible at $24 \mathrm{~h}$ after LPS challenge; neutrophils were found extravasating from blood vessels specifically in the LPS hemisphere. PXS-4681A decreased the number of
MPO-positive cells in the striatum ( $t$-test, $p=0.049)$ (Fig. 2J).

In the striatum ipsilateral to the LPS infusion, the microglia cells, identified with Iba1 marker, showed increased cell counts and cellular hypertrophy, which resulted in an increased Iba1-positive area compared to the contralateral striatum, $24 \mathrm{~h}$ (LPS effect,
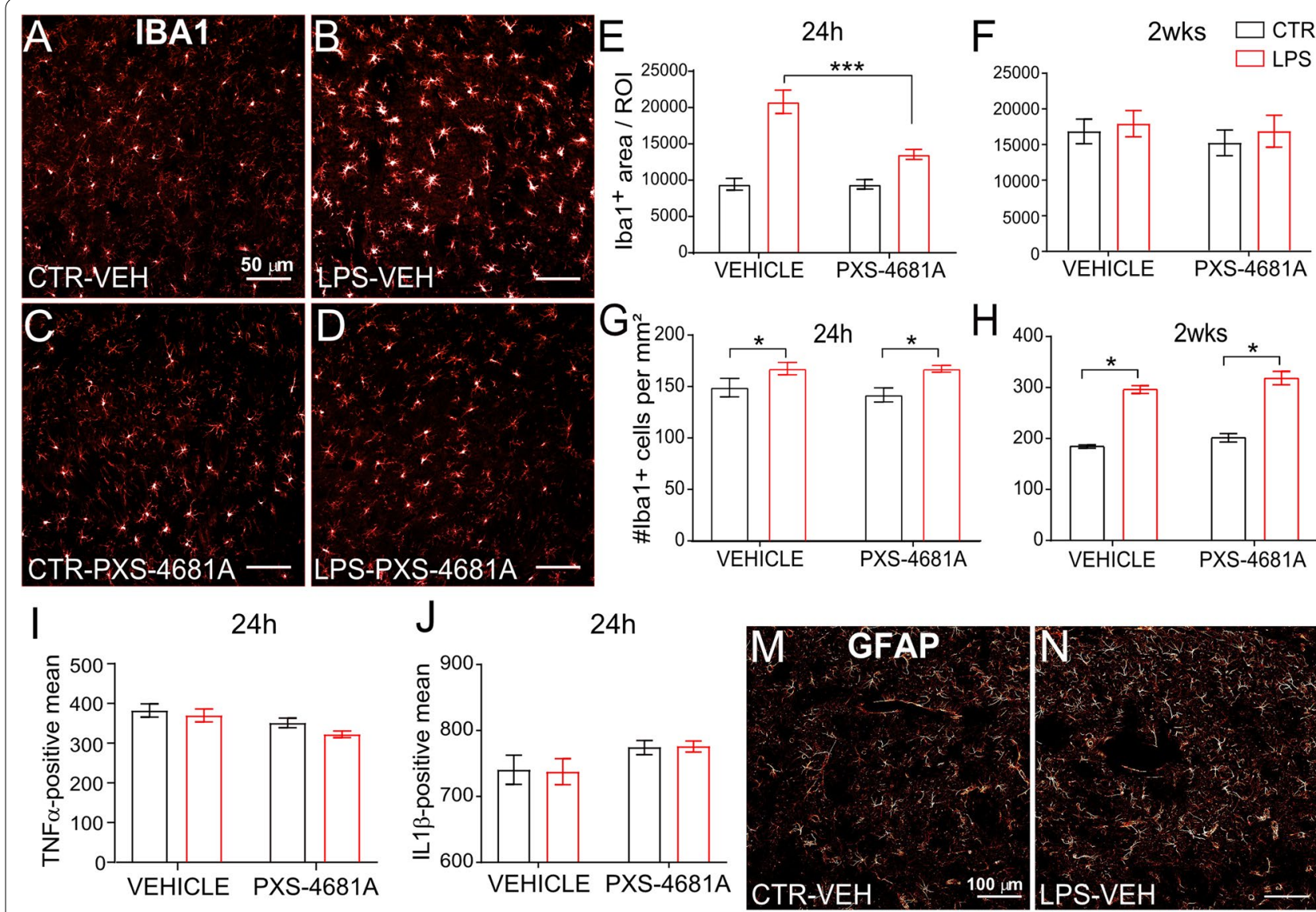

$\mathrm{K}$ $24 \mathrm{~h}$

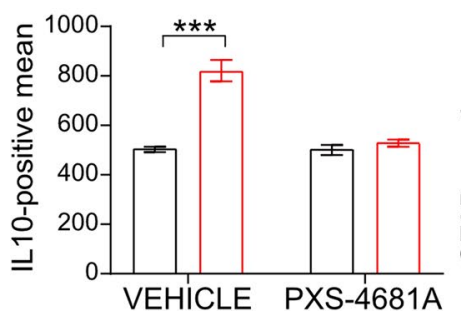

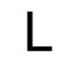

$24 \mathrm{~h}$

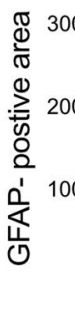

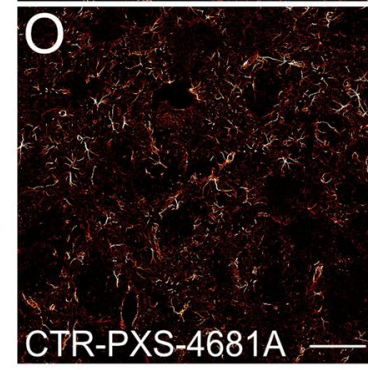

Fig. 3 VAP-1 inhibition reduces the inflammatory response in the striatum. A-D Immunostaining for Iba1 cells in the striatum at 24 h. E Quantification of Iba1-positive area in the ipsilateral striatum (LPS) and contralateral striatum (CTR) at $24 \mathrm{~h}$ after injection of LPS in the SN. LPSVehicle $(n=7)$, LPS-PXS-4681A $(n=5)$. $\mathbf{F}$ Iba1-positive area in the striatum at 2 weeks. Values represent the mean \pm SEM. LPS-Vehicle $(n=10) ;$ LPSPXS4681A $(n=11)$. G Iba1-positive cell count in the striatum at $24 \mathrm{~h}$ after LPS insult. Values represent the mean \pm SEM. $\mathbf{H}$ Iba1-positive cell count in the striatum at 2 weeks. Values represent the mean \pm SEM. I-K Quantification of TNF-alpha, IL-1 beta and IL-10 levels in the striatum at $24 \mathrm{~h}$. Values represent the mean \pm SEM. LPS-Vehicle $(n=7)$, LPS-PXS-4681A $(n=5)$. L Quantification of GFAP-positive area in the dorsal striatum $24 \mathrm{~h}$. LPSVehicle $(n=7)$, LPS-PXS-4681A ( $n=5)$. $\mathbf{M}-\mathbf{P}$ Immunostaining for GFAP in the dorsal striatum. ${ }^{*} p<0.05 ;{ }^{* *} p<0.01,{ }^{* * *} p<0.001$ 
$\left.F_{(1,10)}=85.11\right)$ and 2 weeks (LPS effect, $F_{(1,19)}=6.038$, $p=0.0238$ ) after injection (Fig. 3A-D). This increased cell area was specifically attenuated at $24 \mathrm{~h}$ when the rats received PXS-4681A ip. (Interaction LPS $\mathrm{x}$ treatment, $F_{(1,10)}=16.55, p=0.0028$; Sidak's multiple comparison showed a difference between the two LPS hemispheres at $24 \mathrm{~h}(p=0.0005)$ but not between control hemispheres $(p>0.05)$ (Fig. 3E), nor at 2 weeks (Interaction, $\left.F_{(1,10)}=0.2184\right)$ (Fig. 3F). Microglia numbers increased in the striatum at both time points, resisting PXS-4681A (Fig. 3G-H): two-way repeated measures ANOVA revealed an effect of LPS, $F_{(1,10)}=8.343, p=0.0162$; but neither an effect of PXS-4681A, $F_{(1,10)}=0.2836$, nor a significant interaction, $F_{(1,10)}=0.2184$, at $24 \mathrm{~h}$ timepoint; and an effect of LPS, $F_{(1,19)}=102.9, p<0.0001$; but neither an effect of PXS-4681A, $F_{(1,19)}=0.8767$, nor a significant interaction, $F_{(1,19)}=0.1088$, at 2 weeks after LPS.

Once activated, microglia undergo several key morphological changes including the uptake of MHC-II protein, and secretion of pro-inflammatory signalling molecules, detrimental to DA neurons [61, 62]. MHCII expression, which plays a key role in $\mathrm{CD} 4+\mathrm{T}$ cell activity [63, 64], increased at 2 weeks after LPS infusion (LPS effect $F_{(1,19)}=6.985, p=0.0165$ ) (Additional file 1: Fig. S1). VAP-1 inhibition did not affect this expression, suggesting that VAP-1 does not influence the CD4 response in the brain after LPS insult; PXS-4681A effect $F_{(1,19)}=0.0075, \quad p>0.05 ; \quad$ Interaction $F_{(1,19)}=0.08489$, $p>0.05$.

We detected an overall low level of pro-inflammatory cytokines such as tumour necrosis alpha (TNF)-alpha and interleukin (IL)-1beta in the striatum. TNF-alpha was found to be decreased in the striatum in the presence of LPS (Fig. 3I), LPS effect $F_{(1,10)}=24.77, p=0.001$, whereas IL-1beta was unaffected $\left(F_{(1,10)}=0.006\right)$ (Fig. 3J). No main effect of PXS-4681A nor an interaction was detected for either cytokine. At the same time, IL-10 increased after LPS infusion in the ipsilateral hemisphere compared to the control hemisphere, LPS effect $F_{(1,10)}=22.92$, $p<0.001$. This effect was absent in the PXS-4681A group, PXS-4681A effect $F_{(1,10)}=18.15, p=0.0017$, producing a significant interaction $F_{(1,10)}=16.61, p=0.0022$ : there was no difference between LPS and control hemispheres (significant difference between control and LPS hemisphere in the LPS-Vehicle group $(p<0.001)$, but not in the LPS-PXS-4681A group $(p>0.05))$ (Fig. 3K).

As for microglia, striatal astrocytes also react to the LPS stimulus. Analysis of the GFAP-positive area in the dorsal striatum found an increase in response to the LPS infusion, $F_{(1,10)}=22.75, p=0.008$. Inhibition of VAP-1 decreased the LPS-induced response: there was no effect of PXS-4681A, $F_{(1,10)}=1.468$, but a significant LPS $\mathrm{x}$ PXS-4681A interaction was detected, $F_{(1,10)}=7.482$, $p=0.021$, indicating that there was a significant difference between control and LPS hemispheres in the Vehicle group $(p=0.0003)$ but not in the PXS-4681A group (Fig. 3L-P).

Severe nigrostriatal dopamine depletion and inflammatory response are both associated with postsynaptic changes on striatal spiny projection neuron morphology and modulation of striatal synaptic plasticity [65]. Two weeks after LPS challenge, we found LPS had no effect on the intensity of PSD-95-positive staining; LPS effect $F_{(1,23)}=0.3449, p>0.05$ (Additional file 1: Fig. S2A-D) but PXS-4681A was able to increase the intensity of PSD-95-positive puncta in both hemispheres as compared to Vehicle-treated rats $\left(F_{(1,23)}=6.060, p=0.0218\right)$ (Additional file 1: Fig. S2E). No Interaction $F_{(1,23)}=1.158$, $p>0.05$ was found and no effect in the number of PSD95 puncta induced by either LPS or PXS-4681A was observed.

\section{VAP-1 inhibition restores habit learning induced by a loss of DA neurons in SN}

In addition to motor impairment, loss of DA neurons in the lateral $\mathrm{SN}$ is associated with a loss of habit learning $[66,67]$ leading to a diminished ability to transfer routine activities to a less resource intensive process, increasing cognitive load and inducing cognitive interference. We sought to investigate the effect of VAP-1 inhibition on the impact of intranigral LPS on habit learning. LPS was injected bilaterally into the lateral SN (Fig. 4A), resulting in the disruption of the DA projection to the dorsolateral striatum (DLS) (Fig. 4C and C'). Goal-directed actions require the encoding of action-outcome $(\mathrm{A}-\mathrm{O})$ associations but, over the course of extended training, control over these actions shifts from a goal-directed process to a habitual process, the latter relying on the formation of stimulus-response $(\mathrm{S}-\mathrm{R})$ associations, rendering them

(See figure on next page.)

Fig. 4 VAP-1 inhibition reverses the deficit in habitual motor learning. A LPS infusion. B Behavioural task. C-C'TH-immunoreactivity in the striatum $\mathbf{C}$ and in the SN $\left.\mathbf{C}^{\prime}\right)$ for the LPS-injected animals. D Lever pressing rate during training. Saline $(n=16)$, LPS-Vehicle $(n=21)$, LPS-PXS-4681 A $(n=17)$. E Extinction test. The mean percentage as compared to baseline lever press rate for the devalued and the valued groups is shown. Saline-Vehicle devalued $(n=8)$, valued ( $n=8)$; LPS-Vehicle devalued $(n=10)$, valued $(n=11)$; LPS-PXS-4681A devalued $(n=8)$, valued $(n=9)$. F Consumption test on day 3 of taste aversion devaluation. G Grip Strength Meter. $\mathbf{H}$ Rotarod performance. I Average of lever press rate of the two outcomes during training. Saline-Vehicle $(n=6)$; LPS-Vehicle $(n=6)$; LPS-PXS-4681A $(n=6)$. J Mean lever press rate during extinction test after specific satiety devaluation. Values represent the mean \pm SEM. ${ }^{*} p<0.05 ;{ }^{* *} p<0.01,{ }^{* * *} p<0.001$ 


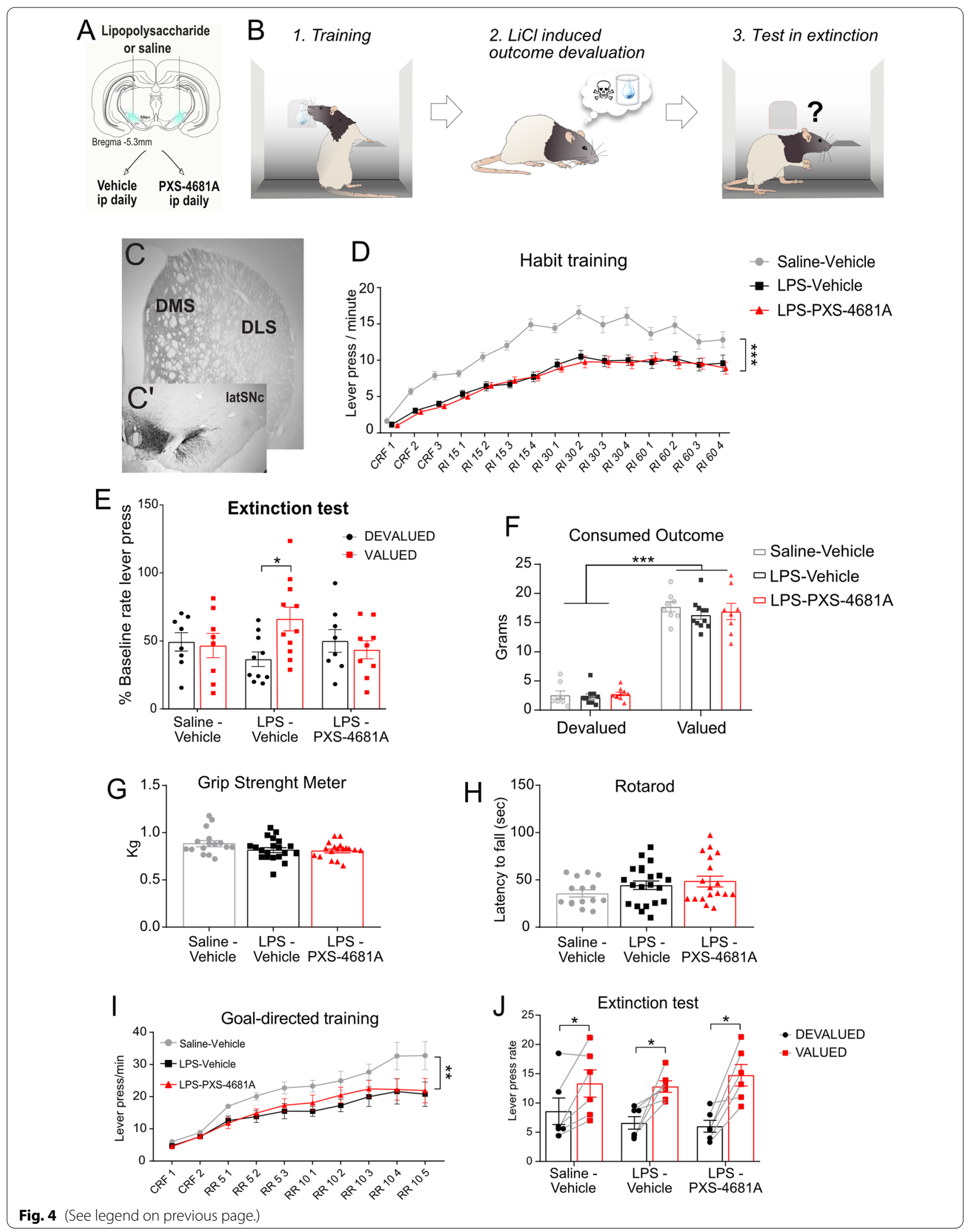


insensitive to changes in outcome value, something that is usually demonstrated using an outcome devaluation test [68-70].

Two weeks after LPS was bilaterally infused into the lateral $\mathrm{SN}$, food-restricted rats started 15 sessions of instrumental training (overtraining) to press a lever for a sucrose reward delivered on increasing random interval (RI) schedules (Fig. 4B and D). Groups were trained until they earned the same number of sucrose outcomes, so that they were exposed to the same number of reinforced lever presses. After training, the sucrose reward was devalued using $\mathrm{LiCl}$-induced conditioned taste aversion for half the experimental group (using methods described in Lingawi and Balleine [71]) (devalued in Fig. 4E), whereas for the remainder it was not devalued (valued in Fig. 4E).

We anticipated that overtraining would induce habit formation and so reduce sensitivity to outcome devaluation in the Vehicle control group (i.e. valued=devalued) and this is what we found (Saline-Vehicle group in Fig. 4E). In contrast, bilateral infusion of LPS into the SN was predicted to produce a deficit in habit formation rendering lever pressing sensitive to outcome devaluation in the LPS-Vehicle group, which again was confirmed (LPS-Vehicle group in Fig. 4E). Importantly, VAP-1 inhibition by PXS-4681A rescued this deficit in habit learning, blocking the effects of LPS and generating the predicted insensitivity to outcome devaluation in the LPS-PXS-4681A group (Fig. 4E); ANOVA revealed a significant group $\mathrm{x}$ devaluation interaction $F_{(2,48)}=3.617, p=0.0344$; Sidak's multiple comparisons test found a significant devaluation effect in the LPSVehicle group, $p=0.0143$, but no devaluation effect in either the Saline-Vehicle and LPS-PXS-4681A groups, $p>0.05$. The consumption of reinforcers during devaluation differed significantly between the devalued and valued groups (Fig. 4F), $F_{(1,48)}=491$, but there were no differences among the devalued groups, which showed a similar taste aversion effect. A detailed analysis of performance during training indicated that the mean rate of lever pressing progressively increased for all groups $\left(F_{(4.681,238.7)}=124.2\right)$. However, LPS lesions produced an overall lower lever press rate compared to SalineVehicle (Group difference $F_{(2,51)}=20.67$, Interaction $\left.F_{(28,714)}=3.540, \quad p>0.001\right)$ : Tukey's multiple comparisons showed a significant difference between Saline and LPS-Vehicle groups $(p<0.0001)$, a significant difference between Saline and LPS-PXS-4681A $(\mathrm{p}<0.0001)$ and no difference between LPS-Vehicle and LPS-PXS-4681A groups (Fig. 4D). As with the general failure to observe an effect on healthy tissue, there was, therefore, no clear evidence suggesting that PXS-4681A had an additional effect on performance to LPS, although this cannot be definitely confirmed. This decreased lever press performance in the LPS groups was not a result of impaired motor coordination or balance as neither rotarod nor grip strength meter tests showed differences between groups (Fig. 4G, H). Specifically, one-way ANOVA showed no group effect for rotarod, $F_{(2,51)}=1.726$, $p>0.05$, and no group effect for grip strength meter, $F_{(2,51)}=2.497, p>0.05$.

\section{LPS infusion into lateral SN and VAP-1 inhibition do not affect goal-directed action}

It is conceivable that LPS blocked habit learning but that PXS-4681A enhanced goal-directed learning in a way unrelated to the LPS effect. To test this hypothesis, at the end of habit training and testing, rats were given sessions of training on two lever press actions with two different types of outcome (purified or grain pellets, counterbalanced), delivered on random ratio (RR) schedules to assess whether the groups differentially acquired new goal-directed actions. This training was followed by an outcome devaluation test which, following prior procedures-and to minimize any generalized effects of taste aversion treatment, used specific satiety-induced outcome devaluation [69]. All groups learned the new A-O contingencies, showing increased mean lever press rates over the course of training, $F_{(9,135)}=58.95$, $p<0.0001$, although the LPS lesion again reduced lever press rate compared to control $\left(F_{(2,15)}=3.597, p=0.001\right.$; Fig. 4I). Nevertheless, if the new $\mathrm{A}-\mathrm{O}$ associations were properly encoded, the animals should choose the action associated with the still valued outcome over the action associated with the now devalued outcome. Importantly, we found no effect of either LPS, PXS-4681A, or their interaction on the sensitivity of the lever press actions to outcome devaluation and a reliable devaluation effect was observed in each experimental group (Fig. 4J): two-way ANOVA showed an effect of devaluation, $F_{(1,15)}=68.74, p<0.0001$ but no Interaction between groups, $F_{(2,15)}=1.360$. Sidak's multiple comparisons showed a significant difference between devalued and valued actions in each group $(p<0.05)$. As such, these results suggest that the influence of LPS-induced dopamine neuron degeneration in the lateral SN was specific to habit learning and the rescue of habit learning by the VAP-1 inhibitor was not secondary to an off-target influence on goal-directed action.

\section{Discussion}

VAP-1 has been extensively studied in the past for its role in mediating the infiltration of leukocytes in response to inflammatory stimuli [18]. In the brain, VAP-1 is expressed on the $\mathrm{BBB}$ endothelium; in inflammatory conditions, endothelial cells increase their expression 
of adhesion molecules, determining the type of leukocyte infiltrating the tissue [36]. Importantly, in the current series of experiments we found evidence of VAP-1 expression in SN capillaries and choroid plexus at the ventricles and we demonstrated that inhibiting VAP-1 not only targeted neutrophil extravasation, but also indirectly protected the $\mathrm{BBB}$ and attenuated both microglia and astrocyte responses at early time points. Furthermore, when the anti-inflammatory effect was lost at 2 weeks post-LPS, long after the neutrophil infiltration peak, the protective effect on neuronal function was still present, whereas there was no effect on microglia response, again suggesting that VAP-1 inhibition does more than simply affect the neutrophil response.

Our finding that VAP-1 inhibition protected DA neurons from degeneration and rescued behavioural function can be explained as a down-stream effect of an earlier modulation of neutrophil extravasation, as well as a consequence of the inhibition of SSAO enzymatic activity. In fact, VAP-1 is not only an adhesion protein; it also carries an enzymatic moiety that is widely diffused in the blood, with by-products such as ammonia, $\mathrm{H}_{2} \mathrm{O}_{2}$, formaldehyde and methylglyoxal increasing oxidative stress, vascular degeneration and protein unfolding [7274]. Moreover, $\mathrm{H}_{2} \mathrm{O}_{2}$ has been implicated as an underlying factor in the initiation and progression of Parkinson's disease. Increases in endogenous $\mathrm{H}_{2} \mathrm{O}_{2}$ in the dorsal striatum attenuated electrically evoked DA release and also decreased basal DA levels [75]. Indirectly, or at later stages [76], VAP-1 derived end-products also activate the endothelium, up-regulating E-selectin, chemokine CXCL8, vascular and intracellular adhesion molecule 1 (VCAM-1 and ICAM-1) resulting in increased lymphocyte adhesion [77]. It is plausible, therefore, that, even in the absence of a modulation of microglia response at 2 weeks, the inhibition of VAP-1 protects DA neurons by decreasing the production of ROS and vascular oxidative stress.

When injected into the SN, LPS induces an acute inflammatory response. The LPS-activated microglia and neutrophils release pro-inflammatory and neurotoxic factors such as IL-1, TNF-alpha, IL-6, NO and ROS that then cause neuronal damage $[78,79]$, suggesting that neuronal death is secondary to the inflammatory response [60]. Subsequently, the damaged neurons release neuromelanin and abnormal alpha-synuclein as injury signals to prompt reactive microgliosis [80-82] The activation of microglia, via nicotinamide adenosine dinucleotide phosphate (NADPH) oxidase and toll-like receptor (TLR)-4, leads to further production of ROS and pro-inflammatory cytokines [83], inducing a self-amplifying cycle, which then results in a chronic inflammatory response and progressive DA neurodegeneration. In line with this hypothesis, an epidemiological study illustrated that the risk of developing Parkinson's disease is significantly reduced by regular use of non-steroidal antiinflammatory drugs such as ibuprofen [84].

It is not clear, however, whether intranigral LPS induces a chronic inflammatory response, mimicking the conditions under which microglia and neutrophils are activated in neurodegenerative diseases; nevertheless, it does recapitulate many features of Parkinson's disease. First, DA neurons show a particular vulnerability to LPS-induced neutrophil infiltration [85, 86]; LPS is neurotoxic only in the presence of microglia $[87,88]$; when injected into the striatum, LPS induces a progressive neuronal death over a 4-week period together with decreased motor performance on rotarod [89] and the accumulation of alphasynuclein and ubiquitin [90]. These motor abnormalities can be improved by the administration of levodopa [89]. We suggest, therefore, that LPS can provide a useful tool to investigate the effect of the inflammatory process on DA neurons and to assess new anti-inflammatory drugs, particularly in conditions where an acute inflammatory response and neutrophil infiltration are observed. However, with intranigral LPS injections, the progression of the DA loss is relatively fast, limiting the study to a neuroprotective approach.

As with other toxins that are used to induce DA neuronal death, stereotaxic injection of LPS reduces the activity of mitochondrial complex I of the electron transport chain in both SN and striatum [90], similar to what is observed in Parkinson's patients [91]. The mechanism is associated with the upregulation of NADPH oxidase, essential to the production of superoxide [90, 92], and detrimental to DA neurons [92].

LPS direct injection also downregulates superoxide dismutase and glutathione peroxidase, two important antioxidant enzymes [93], whose absence can exacerbate neuronal dysfunction and death [92]. Currently, there is no clear evidence that SSAO/VAP-1 inhibition can directly modulate these enzymes; however, aminoguanidine, an SSAO inhibitor, has been shown to reduce nitric oxide synthase [94, 95], which normally leads to the production of superoxide at the expense of NADPH. In addition, augmented SSAO immunoreactivity appears to be associated with elevated $\mathrm{Cu} / \mathrm{Zn}$ superoxide dismutase 1 expression in blood vessels of Alzheimer's disease brains [26], possibly to protect cells from the SSAOreleased superoxide. Indeed, the plasma of patients with sporadic Alzheimer's disease at moderate-severe and severe stages, has shown increased activity from circulating SSAO/VAP-1, compared to healthy controls [96]. These results highlight the importance of the circulating enzyme, which possibly derives from the shedding of the membrane-bound VAP-1, and results in increased ROS 
production not only at the site of inflammation, but also systemically, which can further contribute to oxidative stress and vascular damage. We are, therefore, supportive of the hypothesis that the neuronal protection provided by the inhibition of SSAO/VAP-1 in the current study comes from both a local decrease in the infiltration of neutrophils and ROS production as well as from a more systemic effect in the blood stream, which prevents vascular dysfunction and downstream effects. However, the specific mechanism of this latter effect must await future experiments.

When injected into the SN, LPS binds to the TLR-4 expressed on endothelial cells and on microglia but not on astrocytes [97]. Within $24 \mathrm{~h}$ from the injection, the microglia population disappears [85], leaving the endothelium to drive the inflammatory cascade, which allows the infiltration of neutrophils and primes the astrocytic response in the $\mathrm{SN}$. An interaction between activated endothelium and astrocytes is also evident in models of Parkinson's disease [58] and in Alzheimer's disease patients, where there is an increase of reactive astrocytes in vessels with a higher expression of VAP-1 [25].

In the striatum LPS resulted in fewer neutrophils infiltrating and adhering to vessels compared to $\mathrm{SN}$ and both astrocytes and microglia cells increased their signature markers at $24 \mathrm{~h}$ post-LPS. The synchronous response of these two cell types suggests they may be acting interdependently and, as observed in LPS systemic models and Alzheimer's disease [97-99], LPS exposure promotes the M1 microglia phenotype that consequently elicits the shift to an A1 phenotype in astrocytes. However, at $24 \mathrm{~h}$ after LPS neither IL-1beta nor TNF-alpha were significantly increased in the striatum, whereas they have been reported to increase in the SN [100]. It is possible that in the striatum pro-inflammatory cytokines peak earlier [60] or for a shorter period and that at $24 \mathrm{~h}$ only resolving cytokines, such as IL-10, are released [101]. IL-10, generally known as an anti-inflammatory cytokine, is increased in the striatum. This is not surprising as it peaks later than pro-inflammatory cytokines. However, recent research has established that IL-10 can be released by neutrophils [102] as a restorative mechanism after interaction with LPS-stimulated Treg cells and that IL-10 promotes apoptosis in monocytes/macrophages [103] and in neutrophils [104].

The spiny projecting neuron is the target of the dopamine innervation of the striatum, and comprises more than $90 \%$ of striatal neurons. In Parkinson patient's, striatal DA denervation correlates with striatal spine loss on spiny projecting neurons and remodelling of axospinous glutamatergic synapses. Loss of striatal spines is an early event that does not correlate with the severity of motor symptoms, suggesting that might be a compensating adaptation for the loss of striatal DA innervation in the early stages of the disease [105]. Both post-mortem idiopathic Parkinson's patients and animal models using 6-OHDA and MPTP, show loss of dendritic spines induced by striatal dopamine depletion [106-109]. We quantified postsynaptic puncta of medium spiny neurons in the dorsal striatum using PSD-95-positive staining and observed that the LPS lesion did not affect the intensity of this staining. PSD-95, important for restoring physiological synaptic functioning, is upregulated by the VAP-1 inhibitor and its restoration is possibly important to ameliorate brain function. However, more investigation on this specific aspect is required.

Several new anti-inflammatory drugs are now in clinical trials for Parkinson's disease [110, 111]. However, due to the complexity of the disease and the interactions of different mechanisms, design of a successful intervention to protect the nigrostriatal pathway will likely require a multi-target approach initiated during the earliest stages of disease. This is particularly important because inflammation is considered an early event in the progression of Parkinson's disease and other neurodegenerative diseases and early diagnosis is key to preventing DA loss. In this regard, specific analysis of inflammatory markers has been suggested as diagnostic biomarkers [111].

\section{Functional deficits associated with the loss of DA neurons}

Less attention has been given to non-motor symptoms in LPS animal models of Parkinson's disease and, considering that the onset of non-motor symptoms precedes the onset of motor symptoms, it is important to understand non-motor aspects. In the current study, we found that a specific effect of LPS infusion into lateral SN was a loss of habit learning. Blockade of the VAP-1 receptor after LPS infusion reduced the neuropathological hallmarks of the insult and rescued the learning deficit caused by the loss of DA terminals in the DLS. Dopamine loss in the striatum is not only associated with motor impairments, but also with cognitive decline. The loss of DA neurons in Parkinson's disease patients starts in the lateral part of the $\mathrm{SN}$ and worsens as the condition endures [67]. The lateral SN projects to the sensorimotor putamen [112], which is implicated in decision-making, particularly in the control of habitual actions [113, 114]. When making a choice amongst several alternatives, we usually deliberate over the consequences of actions and then select an action based on its value. This process is called goaldirected decision-making [115]. Under invariant conditions, however, actions can become more automatic as they come under habitual control. In Parkinson's disease patients, learning and performance processes subserving habitual action control are impaired, forcing patients to maintain cognitive control of even very commonly 
performed actions, resulting in deficits in executing fast, automatic actions.

We found that bilateral LPS injections into the lateral SN disrupted the influence of habit learning on instrumental performance, and that treatment with PXS4681A reversed this deficit. This is relevant because, despite producing only a partial $\sim 20 \%$ protection from neuronal loss and the ability to control the inflammatory response only in the early stages after the insult, PXS-4681A potently rescued this important behavioural function. Furthermore, PXS-4681A was neuroprotective without affecting the function of other brain areas; we found, for example, that goal-directed actions-which are mediated by the dorsomedial striatum [116]-were unaffected by its administration. We speculate that, by decreasing the inflammatory response in the $\mathrm{SN}$ and striatum at early time points, PXS-4681A rescued DA loss and decreased the neuronal stress induced by the inflammatory response. This also resulted in the restoration of cognitive and motor functions affected by the loss of dopamine. The results of this study suggest, therefore, that VAP-1 inhibition may be a relevant target for protecting DA neurons from acute inflammatory stimuli and subsequent cell death and indicate that VAP-1 inhibition is likely to be particularly effective in reducing the effects of inflammatory responses in the $\mathrm{SN}$ in situations where the neutrophilic component is of relevance.

\begin{abstract}
Abbreviations
BBB: Blood-brain barrier; CRF: Continuous reinforcement; DA: Dopaminergic; DAB: 3,3'-Diaminobenzidine; DLS: Dorsolateral striatum; GFAP: Glial fibrillary acidic protein; Iba1: Ionized calcium binding adaptor molecule 1; ICV: Intracerebroventricular; IL: Interleukin; IP: Intraperitoneal; LPS: Lipopolysaccharide; MHC: Major histocompatibility complex; MPO: Myeloperoxidase; NADPH: Nicotinamide adenosine dinucleotide phosphate; PBS: Phosphate buffer saline; PFA: Paraformaldehyde; PSD-95: Postsynaptic density protein 95; RECA: Rat endothelial cell antibody; RI: Random interval; RR: Random ratio; SN: Substantia nigra; SSAO: Semicarbazide-sensitive amine oxidase; TH: Tyrosine hydroxylase; TNF: Tumour necrosis factor; VAP-1: Vascular adhesion protein 1.
\end{abstract}

\section{Supplementary Information}

The online version contains supplementary material available at https://doi. org/10.1186/s12974-021-02288-8.

Additional file 1. Figure S1. MHC-II expression in striatal microglia. Figure S2. VAP-1 inhibition increases postsynaptic protein in the striatum.

\section{Acknowledgements}

The authors thank Wolfgang Jarolimek and Jonathan Foot (Pharmaxis Ltd) for their comments on a draft of the manuscript and for providing PXS-4681 A.

\section{Authors' contributions}

$S B, A B$ and BWB conceived this project. SB performed the experiments, collected and analysed the data under the supervision of BWB. SB and BWB wrote the manuscript and $A B$ revised it. All authors read and approved the final manuscript.

\section{Funding}

This research was supported by grants from the Australian Research Council, DP200103401, DP160105070, an Investigator Grant from the National Health and Medical Research Council of Australia, GNT1175420, to BWB.

\section{Availability of data and materials}

The datasets used and/or analysed during the current study are available from the corresponding author on reasonable request.

\section{Declarations}

\section{Ethics approval and consent to participate}

All of the procedures in these experiments were performed according to the protocol 2013/5960 approved by the Animal Ethics Committee of the University of Sydney, which complies with the National Health and Medical Research Council of Australia code of practice for the care and use of animals for scientific purposes.

\section{Consent for publication}

Not applicable.

\section{Competing interests}

The authors declare no conflict of interest.

\section{Author details}

${ }^{1}$ Decision Neuroscience Lab, School of Psychology, UNSW Sydney, Randwick, NSW 2052, Australia. ${ }^{2}$ Aegros Pharmaceuticals, Sydney, Australia.

Received: 7 July 2021 Accepted: 4 October 2021

Published online: 15 October 2021

\section{References}

1. Skaper SD, Facci L, Zusso M, Giusti P. An inflammation-centric view of neurological disease: beyond the neuron. Front Cell Neurosci. 2018. https://doi.org/10.3389/fncel.2018.00072.

2. Tansey MG, Frank-Cannon TC, McCoy MK, Lee JK, Martinez TN, McAlpine $\mathrm{FE}$, et al. Neuroinflammation in Parkinson's disease: is there sufficient evidence for mechanism-based interventional therapy? Front Biosci. 2008;13:709-17.

3. Abbott NJ. Inflammatory mediators and modulation of blood-brain barrier permeability. Cell Mol Neurobiol. 2000;20:131-47.

4. Huber JD, Egleton RD, Davis TP. Molecular physiology and pathophysiology of tight junctions in the blood-brain barrier. Trends Neurosci. 2001;24(12):719-25.

5. Borlongan CV, Glover LE, Sanberg PR, Hess DC. Permeating the blood brain barrier and abrogating the inflammation in stroke: implications for stroke therapy. Curr Pharm Des. 2012;18(25):3670.

6. Bennett J, Basivireddy J, Kollar A, Biron KE, Reickmann P, Jefferies WA, et al. Blood-brain barrier disruption and enhanced vascular permeability in the multiple sclerosis model EAE. J Neuroimmunol. 2010;229(1-2):180-91.

7. Felger JC, Treadway MT. Inflammation effects on motivation and motor activity: role of dopamine. Neuropsychopharmacology. 2017;42(1):21641. https://doi.org/10.1038/npp.2016.143.

8. Zlokovic BV. Neurovascular pathways to neurodegeneration in Alzheimer's disease and other disorders. Nat Rev Neurosci. 2011. https://doi. org/10.1038/nrn3114.

9. Solé M, Esteban-Lopez M, Taltavull B, Fábregas C, Fadó R, Casals N, et al. Blood-brain barrier dysfunction underlying Alzheimer's disease is induced by an SSAONAP-1-dependent cerebrovascular activation with enhanced A $\beta$ deposition. Biochim Biophys Acta - Mol Basis Dis. 2019. https://doi.org/10.1016/j.bbadis.2019.04.016.

10. Rossi B, Angiari S, Zenaro E, Budui SL, Constantin G. Vascular inflammation in central nervous system diseases: adhesion receptors controlling leukocyte-endothelial interactions. J Leukoc Biol. 2011;89(4):539-56.

11. Kortekaas R, Leenders KL, van Oostrom JCH, Vaalburg W, Bart J, Willemsen ATM, et al. Blood-brain barrier dysfunction in parkinsonian midbrain 
in vivo. Ann Neurol. 2005;57(2):176-9. https://doi.org/10.1002/ana. 20369.

12. Barcia C, Emborg ME, Hirsch EC, Herrero M-T. Blood vessels and parkinsonism. Front Biosci. 2004;9:277-82.

13. Desai BS, Monahan AJ, Carvey PM, Hendey B. Blood-brain barrier pathology in Alzheimer's and Parkinson's disease: implications for drug therapy. Cell Transplant. 2007:16(3):285-99.

14. Fu Q, Song R, Yang Z, Shan Q, Chen W. 6-Hydroxydopamine induces brain vascular endothelial inflammation. IUBMB Life. 2017;69(11):88795. https://doi.org/10.1002/iub.1685.

15. Carvey PM, Zhao CH, Hendey B, Lum H, Trachtenberg J, Desai BS, et al. 6-Hydroxydopamine-induced alterations in blood-brain barrier permeability. Eur J Neurosci. 2005;22(5):1158-68. https://doi.org/10.1111/j. 1460-9568.2005.04281.X.

16. Sarkar S, Raymick J, Mann D, Bowyer JF, Hanig JP, Schmued LC, et al. Neurovascular changes in acute, sub-acute and chronic mouse models of Parkinson's disease. Curr Neurovasc Res. 2014;11(1):48-61.

17. Muller WA. Leukocyte-endothelial cell interactions in the inflammatory response. Lab Investig. 2002;82(5):521-33.

18. Salmi M, Jalkanen S. A 90-kilodalton endothelial cell molecule mediating lymphocyte binding in humans. Science. 1992;257(5075):1407-9.

19. Salmi M, Jalkanen S. VAP-1: an adhesin and an enzyme. Trends Immunol. 2001;22(4):211-6.

20. Smith DJ, Salmi M, Bono P, Hellman J, Leu T, Jalkanen S. Cloning of vascular adhesion protein 1 reveals a novel multifunctional adhesion molecule. J Exp Med. 1998;188(1):17.

21. Jalkanen S, Salmi M. VAP-1 and CD73, endothelial cell surface enzymes in leukocyte extravasation. Arterioscler Thromb Vasc Biol. 2008;28(1):18-26.

22. Tohka S, Laukkanen M, Jalkanen S, Salmi M. Vascular adhesion protein 1 (VAP-1) functions as a molecular brake during granulocyte rolling and mediates recruitment in vivo. FASEB J. 2001;15(2):373-82.

23. Yu PH. Deamination of methylamine and angiopathy; toxicity of formaldehyde, oxidative stress and relevance to protein glycoxidation in diabetes. In: Finberg JPM, Youdim MBH, Riederer P, Tipton KF, editors. The mother of all amine oxidases. Vienna: Springer; 1998.

24. Jaakkola K, Nikula T, Holopainen R, Vähäsilta T, Matikainen MT, Laukkanen $\mathrm{ML}$, et al. In vivo detection of vascular adhesion protein-1 in experimental inflammation. Am J Pathol. 2000;157(2):463-71.

25. Valente T, Gella A, Solé M, Durany N, Unzeta M. Immunohistochemical study of semicarbazide-sensitive amine oxidase/vascular adhesion protein-1 in the hippocampal vasculature: pathological synergy of Alzheimer's disease and diabetes mellitus. J Neurosci Res. 2012;90(10):1989-96.

26. Ferrer I, Lizcano JM, Hernández M, Unzeta M, Herna M. Overexpression of semicarbazide sensitive amine oxidase in the cerebral blood vessels in patients with Alzheimer's disease and cerebral autosomal dominant arteriopathy with subcortical infarcts and leukoencephalopathy. Neurosci Lett. 2002;321(1-2):21-4.

27. O'Rourke AM, Wang EY, Salter-Cid L, Huang L, Miller A, Podar E, et al. Benefit of inhibiting SSAO in relapsing experimental autoimmune encephalomyelitis. J Neural Transm. 2007;114(6):845-9.

28. Hernandez-Guillamon M, Solé M, Delgado P, García-Bonilla L, Giralt D, Boada C, et al. VAP-1/SSAO plasma activity and brain expression in human hemorrhagic stroke. Cerebrovasc Dis. 2012;33(1):55-63.

29. Ma Q, Manaenko A, Khatibi NH, Chen W, Zhang JH, Tang J. Vascular adhesion protein-1 inhibition provides antiinflammatory protection after an intracerebral hemorrhagic stroke in mice. J Cereb Blood Flow Metab. 2011;31(3):881-93.

30. Watcharotayangul J, Mao L, Xu H, Vetri F, Baughman VL, Paisansathan C, et al. Post-ischemic vascular adhesion protein-1 inhibition provides neuroprotection in a rat temporary middle cerebral artery occlusion model. J Neurochem. 2012;123(Suppl):116-24.

31. Xu H-L, Salter-Cid L, Linnik MD, Wang EY, Paisansathan C, Pelligrino DA. Vascular adhesion protein-1 plays an important role in postischemic inflammation and neuropathology in diabetic, estrogen-treated ovariectomized female rats subjected to transient forebrain ischemia. J Pharmacol Exp Ther. 2006:317(1):19-29.

32. Elo P, Tadayon S, Liljenbäck H, Teuho J, Käkelä M, Koskensalo K, et al. Vascular adhesion protein-1 is actively involved in the development of inflammatory lesions in rat models of multiple sclerosis. J Neuroinflammation. 2018;15(1):1-17.

33. Zenaro E, Pietronigro E, Bianca VD, Piacentino G, Marongiu L, Budui $S$, et al. Neutrophils promote Alzheimer's disease-like pathology and cognitive decline via LFA-1 integrin. Nat Med. 2015;21(8):880-6. https:// doi.org/10.1038/nm.3913.

34. Salmi M, Jalkanen S. Vascular adhesion protein-1: a cell surface amine oxidase in translation. Antioxid Redox Signal. 2019;30(3):314-32.

35. Foot JS, Yow TT, Schilter H, Buson A, Deodhar M, Findlay AD, et al. PXS4681A, a potent and selective mechanism-based inhibitor of SSAO/ VAP-1 with anti-inflammatory effects in vivo. J Pharmacol Exp Ther. 2013:347(2):365-74.

36. Becchi S, Buson A, Foot J, Jarolimek W, Balleine BW. Inhibition of semicarbazide-sensitive amine oxidase/vascular adhesion protein-1 reduces lipopolysaccharide-induced neuroinflammation. Br J Pharmacol. 2017;174(14):2302-17. https://doi.org/10.1111/bph.13832.

37. Castaño A, Herrera AJ, Cano J, Machado A. Lipopolysaccharide intranigral injection induces inflammatory reaction and damage in nigrostriatal dopaminergic system. J Neurochem. 1998;70(4):1584-92.

38. Herrera AJ, Castaño A, Venero JL, Cano J, Machado A. The single intranigral injection of LPS as a new model for studying the selective effects of inflammatory reactions on dopaminergic system. Neurobiol Dis. 2000;7(4):429-47.

39. Bannard C, Leriche M, Bandmann O, Brown CH, Ferracane E, SánchezFerro Á, et al. Reduced habit-driven errors in Parkinson's Disease. Sci Rep. 2019. https://doi.org/10.1038/s41598-019-39294-z.

40. Kilkenny C, Browne WJ, Cuthill IC, Emerson M, Altman DG. Improving bioscience research reporting: the arrive guidelines for reporting animal research. PLoS Biol. 2010;8(6):6-10.

41. Kilkenny C, Browne W, Cuthill IC, Emerson M, Altman DG. Animal research: reporting in vivo experiments: the ARRIVE guidelines. $\mathrm{Br} J$ Pharmacol. 2010;160(7):1577-9.

42. MCGrath JC, Lilley E. Implementing guidelines on reporting research using animals (ARRIVE etc.): New requirements for publication in BJP. Br J Pharmacol. 2015;172(13):3189-93.

43. Paxinos G, Watson C. The rat brain in stereotaxic coordinates, 6th Edition | George Paxinos, Charles Watson | ISBN 9780080475158. 2006.

44. Tomás-Camardiel M, Rite I, Herrera AJ, de Pablos RM, Cano J, Machado $A$, et al. Minocycline reduces the lipopolysaccharide-induced inflammatory reaction, peroxynitrite-mediated nitration of proteins, disruption of the blood-brain barrier, and damage in the nigral dopaminergic system. Neurobiol Dis. 2004:16(1):190-201.

45. Schmidt-Kastner R, Meller D, Bellander BM, Strömberg I, Olson L, Ingvar M. A one-step immunohistochemical method for detection of bloodbrain barrier disturbances for immunoglobulins in lesioned rat brain with special reference to false-positive labelling in immunohistochemistry. J Neurosci Methods. 1993;46(2):121-32.

46. Schallert T, Tillerson JL. Intervention strategies for degeneration of dopamine neurons in Parkinsonism optimizing behavioral assessment of outcome. In: Diseases CNS, editor. Totowa. NJ: Humana Press Inc.; 2000. p. 131-2.

47. Haghdoost-Yazdi H, Sarookhani M, Faraj A, Fraidouni N, Dargahi T. Pharmacology, biochemistry and behavior evaluation of the association between blood homocysteine concentration and the degree of behavioral symptoms in the 6-hydroxydopamine-induced Parkinsonism in rat. Pharmacol Biochem Behav. 2014;124:297-304. https://doi.org/10. 1016/j.pbb.2014.06.020.

48. Ryu JK, McLarnon JG. A leaky blood-brain barrier, fibrinogen infiltration and microglial reactivity in inflamed Alzheimer's disease brain. J Cell Mol Med. 2009. https://doi.org/10.1111/j.1582-4934.2008.00434.x

49. Zipser BD, Johanson CE, Gonzalez L, Berzin TM, Tavares R, Hulette CM, et al. Microvascular injury and blood-brain barrier leakage in Alzheimer's disease. Neurobiol Aging. 2007;28(7):977-86.

50. Gray MT, Woulfe JM. Striatal blood-brain barrier permeability in Parkinson's disease. J Cereb Blood Flow Metab. 2015;35(5):747-50.

51. Liu B, Jiang JW, Wilson BC, Du L, Yang SN, Wang JY, et al. Systemic infusion of naloxone reduces degeneration of rat substantia nigral dopaminergic neurons induced by intranigral injection of lipopolysaccharide. J Pharmacol Exp Ther. 2000;295(1):125-32.

52. Heuer A, Smith GA, Lelos MJ, Lane EL, Dunnett SB. Unilateral nigrostriatal 6-hydroxydopamine lesions in mice I: Motor impairments identify 
extent of dopamine depletion at three different lesion sites. Behav Brain Res. 2012;228(1):30-43. https://doi.org/10.1016/j.bbr.2011.11.027.

53. Miyanishi K, Choudhury ME, Watanabe M, Kubo M, Nomoto M, Yano H, et al. Behavioral tests predicting striatal dopamine level in a rat hemiParkinson's disease model. Neurochem Int. 2019;122:38-46.

54. Lundblad M, Andersson M, Winkler C, Kirik D, Wierup N, Cenci MA. Pharmacological validation of behavioural measures of akinesia and dyskinesia in a rat model of Parkinson's disease. Eur J Neurosci. 2002;15(1):120-32.

55. Creese I, Burt DR, Snyder SH. Dopamine receptor binding enhancement accompanies lesion-induced behavioral supersensitivity. Science. 1977;197(4303):596-8.

56. lancu R, Mohapel P, Brundin P, Paul G. Behavioral characterization of a unilateral 6-OHDA-lesion model of Parkinson's disease in mice. Behav Brain Res. 2005;162(1):1-10.

57. Hamby ME, Sofroniew MV. Reactive astrocytes as therapeutic targets for CNS. Neurotherapeutics. 2010;7(October):494-506.

58. Cabezas R, Ávila M, Gonzalez J, El-Bachá RS, Báez E, García-Segura LM, et al. Astrocytic modulation of blood brain barrier: perspectives on Parkinson's disease. Front Cell Neurosci. 2014. https://doi.org/10.3389/ fncel.2014.00211.

59. Alvarez JI, Katayama T, Prat A. Glial influence on the blood brain barrier. Glia. 2013;61(12):1939-58.

60. Flores-Martinez YM, Fernandez-Parrilla MA, Ayala-Davila J, Reyes-Corona D, Blanco-Alvarez VM, Soto-Rojas LO, et al. Acute neuroinflammatory response in the substantia nigra pars compacta of rats after a local injection of lipopolysaccharide. J Immunol Res. 2018;8(2018):1-19.

61. Nagatsu T, Mogi M, Ichinose H, Togari A. Changes in cytokines and neurotrophins in Parkinson's disease. J Neural Transm Suppl. 2000;60:277-90.

62. Nagatsu T, Sawada M. Inflammatory process in Parkinson's disease: role for cytokines. Curr Pharm Des. 2005. https://doi.org/10.1007/978-3-21145295-0 57.

63. Taylor PR, Martinez-Pomares L, Stacey M, Lin HH, Brown GD, Gordon S. Macrophage receptors and immune recognition. Annu Rev Immunol. 2005;23(1):901-44. https://doi.org/10.1146/annurev.immunol.23. 021704.115816

64. Imamura K, Hishikawa N, Sawada M, Nagatsu T, Yoshida M, Hashizume Y. Distribution of major histocompatibility complex class II-positive microglia and cytokine profile of Parkinson's disease brains. Acta Neuropathol. 2003;106(6):518-26.

65. Picconi B, Gardoni F, Centonze D, Mauceri D, Cenci MA, Bernardi G, et al. Abnormal Ca2+-calmodulin-dependent protein kinase II function mediates synaptic and motor deficits in experimental parkinsonism. J Neurosci. 2004;24(23):5283-91.

66. Pavese N, Brooks DJ. Imaging neurodegeneration in Parkinson's disease. Biochim Biophys Acta Mol Basis Dis. 2009;1792(7):722-9.

67. Redgrave P, Rodriguez M, Smith Y, Rodriguez-Oroz MC, Lehericy S, Bergman $\mathrm{H}$, et al. Goal-directed and habitual control in the basal ganglia: implications for Parkinson's disease. Nat Rev Neurosci. 2010;11(11):760-72

68. Dickinson A. Actions and habits: the development of behavioural autonomy. Philos Trans R Soc London B Biol Sci. 1985. https://doi.org/ 10.1098/rstb.1985.0010.

69. Balleine BW, Dickinson A. Goal-directed instrumental action: contingency and incentive learning and their cortical substrates. Neuropharmacology. 1998;37(4-5):407-19.

70. Balleine BW. The meaning of behavior: discriminating reflex and volition in the brain. Neuron. 2019;104(1):47-62. https://doi.org/10.1016/j. neuron.2019.09.024.

71. Lingawi NW, Balleine BW. Amygdala central nucleus interacts with dorsolateral striatum to regulate the acquisition of habits. J Neurosci. 2012;32(3):1073-81.

72. Boor PJ, Trent MB, Lyles GA, Tao M, Ansari GA. Methylamine metabolism to formaldehyde by vascular semicarbazide-sensitive amine oxidase. Toxicology. 1992;73(3):251-8.

73. Gubisne-Haberle D, Hill W, Kazachkov M, Richardson JS, Yu PH. Protein cross-linkage induced by formaldehyde derived from semicarbazidesensitive amine oxidase-mediated deamination of methylamine. $J$ Pharmacol Exp Ther. 2004;310(3):1125-32.
74. Unzeta M, Hernàndez-Guillamon M, Sun P, Solé M. SSAONAP-1 in cerebrovascular disorders: a potential therapeutic target for stroke and Alzheimer's disease. Int J Mol Sci. 2021. https://doi.org/10.3390/ijms2 2073365.

75. Spanos M, Gras-Najjar J, Letchworth JM, Sanford AL, Toups JV, Sombers LA. Quantitation of hydrogen peroxide fluctuations and their modulation of dopamine dynamics in the rat dorsal striatum using fast-scan cyclic voltammetry. ACS Chem Neurosci. 2013;4(5):782-9.

76. Salmi M, Jalkanen S. Cell-surface enzymes in control of leukocyte trafficking. Nat Rev Immunol. 2005;5(10):760-71.

77. Lalor PF, Sun PJ, Weston CJ, Martin-Santos A, Wakelam MJO, Adams DH. Activation of vascular adhesion protein-1 on liver endothelium results in an NF-KB-dependent increase in lymphocyte adhesion. Hepatology. 2007:45(2):465-74.

78. Deng I, Corrigan F, Zhai G, Zhou X-F, Bobrovskaya L. Lipopolysaccharide animal models of Parkinson's disease: Recent progress and relevance to clinical disease. Brain Behav Immun Heal. 2020;4(March): 100060. https://doi.org/10.1016/j.bbih.2020.100060.

79. Bing G, Liu M. Lipopolysaccharide animal models for Parkinson's disease. Parkinsons Dis. 2011. https://doi.org/10.4061/2011/327089.

80. Zhang X, Xie W, Qu S, Pan T, Wang X, Le W. Neuroprotection by iron chelator against proteasome inhibitor-induced nigral degeneration. Biochem Biophys Res Commun. 2005;333(2):544-9.

81. Zecca L, Wilms H, Geick S, Claasen J-H, Brandenburg L-O, Holzknecht $C$, et al. Human neuromelanin induces neuroinflammation and neurodegeneration in the rat substantia nigra: implications for Parkinson's disease. Acta Neuropathol. 2008:116(1):47-55.

82. Gao HM, Kotzbauer PT, Uryu K, Leight S, Trojanowski JQ, Lee VMY. Neuroinflammation and oxidation/nitration of alpha-synuclein linked to dopaminergic neurodegeneration. J Neurosci. 2008;28(30):7687-98.

83. Zhang Y, Chen F, Chen J, Huang S, Chen J, Huang J, et al. Soyasaponin $\mathrm{Bb}$ inhibits the recruitment of toll-like receptor 4 (TLR4) into lipid rafts and its signaling pathway by suppressing the nicotinamide adenine dinucleotide phosphate (NADPH) oxidase-dependent generation of reactive oxygen species. Mol Nutr Food Res. 2016;60(7):1532-43.

84. Chen H, Jacobs E, Schwarzschild MA, McCullough ML, Calle EE, Thun $\mathrm{MJ}$, et al. Nonsteroidal antiinflammatory drug use and the risk for Parkinson's disease. Ann Neurol. 2005;58(6):963-7.

85. Ji K-A, Eu MY, Kang S-H, Gwag BJ, Jou I, Joe E-H. Differential neutrophil infiltration contributes to regional differences in brain inflammation in the substantia nigra pars compacta and cortex. Glia. 2008;56(10):103947. https://doi.org/10.1002/glia.20677.

86. Ryu JK, Tran KC, McLarnon JG. Depletion of neutrophils reduces neuronal degeneration and inflammatory responses induced by quinolinic acid in vivo. Glia. 2007;55(4):439-51.

87. Gao H-M, Jiang J, Wilson B, Zhang W, Hong J-S, Liu B. Microglial activation-mediated delayed and progressive degeneration of rat nigral dopaminergic neurons: relevance to Parkinson's disease. J Neurochem. 2002;81(6):1285-97.

88. Gibbons HM, Dragunow M. Microglia induce neural cell death via a proximity-dependent mechanism involving nitric oxide. Brain Res. 2006;1084(1):1-15.

89. Hunter RL, Cheng B, Choi D-Y, Liu M, Liu S, Cass WA, et al. Intrastriatal lipopolysaccharide injection induces parkinsonism in C57/B6 mice. J Neurosci Res. 2009:87(8):1913-21.

90. Choi D-Y, Liu M, Hunter RL, Cass WA, Pandya JD, Sullivan PG, et al. Striatal neuroinflammation promotes parkinsonism in rats. Gendelman $\mathrm{HE}$, editor. PLOS ONE. 2009;4(5):e5482.

91. Hauser DN, Hastings TG. Mitochondrial dysfunction and oxidative stress in Parkinson's disease and monogenic parkinsonism. Neurobiol Dis. 2013;51:35-42. https://doi.org/10.1016/..nbd.2012.10.011.

92. Chung ES, Chung YC, Bok E, Baik HH, Park ES, Park J-Y, et al. Fluoxetine prevents LPS-induced degeneration of nigral dopaminergic neurons by inhibiting microglia-mediated oxidative stress. Brain Res. 2010;1363:143-50.

93. Ferreira Mello BS, Monte AS, McIntyre RS, Soczynska JK, Custódio CS, Cordeiro RC, et al. Effects of doxycycline on depressive-like behavior in mice after lipopolysaccharide (LPS) administration. J Psychiatric Res. 2013;47:1521-9. 
94. Corbett JA, Tilton RG, Chang K, Hasan KS, Ido Y, Wang JL, et al. Aminoguanidine, a novel inhibitor of nitric oxide formation, prevents diabetic vascular dysfunction. Diabetes. 1992;41(4):552-6.

95. Yu PH, Zuo DM. Aminoguanidine inhibits semicarbazide-sensitive amine oxidase activity: implications for advanced glycation and diabetic complications. Diabetologia. 1997;40(11):1243-50.

96. del Mar Hernandez M, Esteban M, Szabo P, Boada M, Unzeta M. Human plasma semicarbazide sensitive amine oxidase SSAO beta-amyloid protein and aging. Neurosci Lett. 2005;384(1-2):183-7.

97. Liddelow SA, Guttenplan KA, Clarke LE, Bennett FC, Bohlen CJ, Schirmer $L$, et al. Neurotoxic reactive astrocytes are induced by activated microglia. Nature. 2017;541(7638):481-7. https://doi.org/10.1038/natur e21029.

98. Li K, Li J, Zheng J, Qin S. Reactive astrocytes in neurodegenerative diseases. Aging Dis. 2019;10(3):664-75.

99. Lopategui Cabezas I, Herrera Batista A, Pentón RG. The role of glial cells in Alzheimer disease: potential therapeutic implications. Neurologia. 2014;29(5):305-9.

100. Iravani MM, Leung CCM, Sadeghian M, Haddon CO, Rose S, Jenner $P$. The acute and the long-term effects of nigral lipopolysaccharide administration on dopaminergic dysfunction and glial cell activation. Eur J Neurosci. 2005;22(2):317-30.

101. Lively $S$, Schlichter LC. Microglia responses to pro-inflammatory stimuli (LPS, IFNy+TNFa) and reprogramming by resolving cytokines (IL-4, IL-10). Front Cell Neurosci. 2018. https://doi.org/10.3389/fncel.2018. 00215.

102. Kasten KR, Muenzer JT, Caldwell CC. Neutrophils are significant producers of IL-10 during sepsis. Biochem Biophys Res Commun . 2010;393(1):28-31.

103. Schmidt M, Lügering N, Pauels HG, Schulze-Osthoff K, Domschke W, Kucharzik T. IL-10 induces apoptosis in human monocytes involving the CD95 receptor/ligand pathway. Eur J Immunol. 2000;30(6):1769-77.

104. Lewkowicz N, Mycko MP, Przygodzka P, Ćwiklińska H, Cichalewska M, Matysiak M, et al. Induction of human IL-10-producing neutrophils by LPS-stimulated Treg cells and IL-10. Mucosal Immunol. 2016;9(2):36478. https://doi.org/10.1038/mi.2015.66

105. Villalba RM, Smith Y. Loss and remodeling of striatal dendritic spines in Parkinson's disease: from homeostasis to maladaptive plasticity? J Neural Transm. 2018;125(3):431-47.
106. Day M, Wang Z, Ding J, An X, Ingham CA, Shering AF, et al. Selective elimination of glutamatergic synapses on striatopallidal neurons in Parkinson disease models. Nat Neurosci. 2006:9(2):251-9.

107. Zaja-Milatovic S, Milatovic D, Schantz AM, Zhang J, Montine KS, Samii $A$, et al. Dendritic degeneration in neostriatal medium spiny neurons in Parkinson disease. Neurology. 2005;64(3):545-7.

108. Stephens B, Mueller AJ, Shering AF, Hood SH, Taggart P, Arbuthnott GW, et al. Evidence of a breakdown of corticostriatal connections in Parkinson's disease. Neuroscience. 2005:132(3):741-54.

109. Ingham CA, Hood SH, Arbuthnott GW. Spine density on neostriatal neurones changes with 6-hydroxydopamine lesions and with age. Brain Res. 1989;503(2):334-8.

110. Tansey MG, Goldberg MS. Neuroinflammation in Parkinson's disease: its role in neuronal death and implications for therapeutic intervention. Neurobiol Dis. 2010;37(3):510-8.

111. Pajares M, Rojo IA, Manda G, Boscá L, Cuadrado A. Inflammation in Parkinson's Disease: mechanisms and therapeutic implications. Cells. 2020:9(7):1687.

112. Tremblay P-L, Bedard M-A, Langlois D, Blanchet PJ, Lemay M, Parent M. Movement chunking during sequence learning is a dopaminedependant process: a study conducted in Parkinson's disease. Exp brain Res. 2010;205(3):375-85.

113. Wymbs NF, Bassett DS, Mucha PJ, Porter MA, Grafton ST. Differential recruitment of the sensorimotor putamen and frontoparietal cortex during motor chunking in humans. Neuron. 2012;74(5):936-46.

114. Yin HH, Knowlton BJ, Balleine BW. Lesions of dorsolateral striatum preserve outcome expectancy but disrupt habit formation in instrumental learning. Eur J Neurosci. 2004;19(1):181-9.

115. Balleine BW, O'Doherty JP. Human and rodent homologies in action control: corticostriatal determinants of goal-directed and habitual action. Neuropsychopharmacology. 2010;35(1):48-69.

116. Yin $\mathrm{HH}$, Ostlund SB, Knowlton BJ, Balleine BW. The role of the dorsomedial striatum in instrumental conditioning. Eur J Neurosci. 2005;22(2):513-23.

\section{Publisher's Note}

Springer Nature remains neutral with regard to jurisdictional claims in published maps and institutional affiliations.
Ready to submit your research? Choose BMC and benefit from:

- fast, convenient online submission

- thorough peer review by experienced researchers in your field

- rapid publication on acceptance

- support for research data, including large and complex data types

- gold Open Access which fosters wider collaboration and increased citations

- maximum visibility for your research: over 100M website views per year

At $\mathrm{BMC}$, research is always in progress.

Learn more biomedcentral.com/submissions 